\title{
Conspectus librorum
}

Acosta-HugHes 1996

Acosta-Hughes 2002

AdRAdOS $1990^{3}, 2010^{4}$

AlberTI 1983

AleXANDERSON 1963

AleXANDROU 2016

ALFONSI 1963

Allen 1889

AlPERS 1998

ALy 1909

AMARANTE 1998

ANASTASSIOU-IRMER 1997

ANASTASSIOU-IRMER 2006

ANDORLINI 1993

ANDORLINI 1999
B. Acosta-Hughes, Callimachus, Hipponax and the persona of the iambographer, MD 36, 1996, pp. 205-216.

B. Acosta-Hughes, Polyeideia. The Iambi of Callimachus and the Archaic Iambic Tradition, Berkeley-Los Angeles-London 2002.

Líricos Griegos. Elegiacos y yambógrafos arcaicos, texto trad. por F.R. ADRADOS, I-II, Madrid 1990³ , II $2010^{4}$ (1981²; Barcelona 1956-1959'1).

G.B. Alberti, I papiri e l' "archetipo" di Erodoto, Prometheus 9, 1983, pp. 193-196.

B. AleXANDERSON, Die hippokratische Schrift Prognostikon. Überlieferung und Text (Studia Graeca et Latina Gothoburgensia XVII), Stockholm 1963.

M. Alexandrou, Mytological Narratives in Hipponax, in Iambus and Elegy. New Approaches, Ed. by L. Swift-C. Carey, Oxford 2016, pp. 210-228.

L. Alfonsi, Dagli «Epodi di Strasburgo» a Timocle, Aegyptus 43, 1963, pp. 68-69.

T.W. Allen, Notes on Abbreviations in Greek Manuscripts, Oxford 1889 [rist. in Abbreviations in Greek Inscriptions, Papyri, Manuscripts and Early Printed Book, Compiled by A.N. Oikonomides, Chicago 1974, pp. 131-193].

K. AlPERS, Lexicographica Minora, I: Zu den Fragmenten des Alexander von Kotiaeion, in Dissertatiunculae criticae. Festschrift für Günther Christian Hansen, Würzburg 1998, pp. 93-102.

W. Aly, Ein Beitrag zur Textsgeschichte Herodots, RhM 64, 1909, pp. 591-600.

F. AMARANTE, Eschilo nei papiri ercolanesi, CErc 28, 1998, pp. 139-150.

A. Anastassiou-D. Irmer, Testimonien zum Corpus Hippocraticum, II 1, Gottingen 1997.

A. Anastassiou-D. Irmer, Testimonien zum Corpus Hippocraticum, I, Gottingen 2006.

I. ANDORLINI, L'apporto dei papiri alla conoscenza della scienza medica antica, ANRW II 37.1, 1993, pp. 458-462.

I. ANDORLINI, Riflessi e applicazioni della terapia ippocratica nella testimonianza dei papiri, in Aspetti della terapia del Corpus hippocraticum, "Atti del IX Colloque International Hippocratique" (Pisa 25-29 settembre 1996), a c. di I. 
ANDORLINI 2000

ANDORLINI 2003

ANDORLINI 2008a

ANDORLINI 2008b

ANDORLINI 2009

ANDORLINI-MARCONE 2004

ANDRISANO 1990-1993

Arata-Bastianini-

MONTANARI 2004

ARDIZZONI 1960

ARDIZZONI 1980

ARNOTT 1996

ASCHERI 2010

ASHERI 1988

Austin 1973

AusTin 2008

BACCANI 1989

BAFFIONI 1954

BAFFIONI 1958
Garofalo-A. Lami-D. Manetti-A. Roselli, Aspetti della terapia nel Corpus Hippocraticum, Firenze 1999, pp. 431-446.

I. ANDORLINI, Codici papiracei con scolî e commento, in Le commentaire entre tradition et innovation, "Actes du Colloque international del l'Institut des traditions textuelles", Paris et Villejuif 22-25 septembre 1999 (sous la direction de M.O. Goulet-Cazé), Paris 2000, pp. 37-52.

I. ANDORLINI, L'esegesi del libro tecnico: papiri di medicina con scolî e commento, in Papiri filosofici: miscellanea di studi IV (STCPF 11), Firenze 2003, pp. 9-29.

I. ANDORLINI, Old and New Greek Papyri from Tebtunis in the Bancroft Library of Berkeley: Work in Progress, in GraecoRoman Fayum - Texts and Archaeology, Ed. by S. LippertM. Schentuleit, Wiesbaden 2008, pp. 6-9.

I. ANDORLINI, 4. Aph. III 20, 23-24 (cum scholiis), 29, 31; IV 1 (cum scholiis), 5 (cum scholiis) = PAnt 183, in CPF I.2, Firenze 2008, pp. 89-96.

I. ANDORLINI, Medical Treatise on Prognosis, in I. Andorlini (ed.), Greek Medical Papyri, II, Firenze 2009, pp. 15-33.

I. ANDORLini-A. MARCONE, Medicina, medico e società nel mondo antico, Firenze, 2004.

A. ANDRISANO, Sul primo epodo di Strasburgo (Arch. fr. [79a],7ss. D. ${ }^{3}=$ Hipp. fr. dub. 115,9ss. W. $\left.{ }^{2}\right)$, MCr 25-28, 1990 1993, pp. 23-29.

L. Arata-G. Bastianini-F. Montanari, Aeschylus 3: P.Oxy. XX 2256, in CLGP I.1.1: Aeschines-Alcaeus, München-Leipzig 2004, pp. 34-51.

A. Ardizzoni, Callimaco "Ipponatteo", AFLC 28, 1960, pp. 3-16.

A. Ardizzoni, Riflessioni sul primo giambo di Callimaco (fr. 191 Pfeiffer), GIF 32, 1980, pp. 145-155.

Alexis. The Fragments. A Commentary, by W.G. ARNOTT, Cambridge 1996.

P. AsCHERI, Demetrio Issione 'dialettologo': l'Attico e il dialetto degli Alessandrini, in Aner polytropos. Ricerche di filologia greca antica dedicate dagli allievi a Franco Montanari, a c. di F. Montana, Roma 2010, pp. 125-152.

Erodoto, Le Storie, I: Introduzione generale di D. Asheri, Libro I: La Lidia e la Persia, Testo e commento a cura di D. ASHERI, Milano 1988.

Comicorum Graecorum fragmenta in papyris reperta, ed. C. Austin, Berolini-Novi Eboraci 1973.

C. Austin, Pandore, Prométhée et le mythe des races, in BASTIANINI-CASANOVA 2008, pp. 71-81.

D. BACCANI, Rec. a CPF I.1* , APapyrol 1, 1989, pp. 145-148.

V. BAFFIONI, Inediti di Archelao da un codice bolognese, Bol1Class 3, 1954, pp. 57-76.

V. BAFFIONI, Scoli inediti di Palladio al De sectis di Galeno, Bol1Class 6, 1958, pp. 62-78. 
BAGORDO 2013

BALDWIN 1984

BANDIERA 1997

BARNES 1986

BARNS 1967

BARTOLETTI 1960

BARTOLETTI 1967

BASTIANINI 1992

BASTIANINI 2001

BELL 1922

BELLONI 2000

BERGK $1882^{4}$

BERGSON 1986

BerRueCos Frank 2015

BlASs 1900a

BlAss 1900b

BLASS 1906

BLOUIN 2016
A. BAGordo, Telekleides. Einleitung, Übersetzung, Kommentar (Fragmenta comica 4), Heidelberg 2013.

B. BALDwIN, Herodotus and Tacitus: Two Notes on Ancient Book Titles, QUCC n.s. 16, 1984, pp. 31-34.

A. BANDIERA, Per un bilancio della tradizione papiracea delle Storie di Erodoto, in "Akten des 21. Internationalen Papyrologenkongresses", Berlin 1995, hrsg. von B. Kramer-W. Luppe-H. Maehler-G. Poethke, Berlin 1997, pp. 49-56. J. BARnes, Editor's Notes, Phronesis 31, 1986, pp. 92-100. The Antinoopolis Papyri, III, Ed. with Transl. and Notes by J.W.B. BARNS-H. Zilliacus, London 1967.

V. BARTOLETTI, Tucidide II 73,1-74,1 in un papiro dell'Università Statale di Milano, in Studi in onore di Luigi Castiglioni, Firenze 1960, I, pp. 59-66.

V. BARTOLETTI, P.Mil.Vogl. 205, in Papiri della Università degli Studi di Milano (P. Mil. Vogliano), IV, Milano-Varese 1967, pp. 5-7.

G. BASTIANINI, Le abbreviazioni, in CPF I.1**, Firenze 1992, pp. 276-281 (Hierocles, Elementa moralia, pp. 268-451).

G. BASTIANINI, PMilVogl I 18: perché l'interruzione?, in Studium atque urbanitas, Miscellanea in onore di Sergio Daris, a c. di M. Capasso-S. Pernigotti (Papyrologica Lupiensia 9), Lecce 2001, pp. 76-81.

Esiodo: cent'anni di papiri, "Atti del convegno internazionale di studi", Firenze 7-8 giugno 2007, a c. di G. BASTIANINI-A. CASANOVA, Firenze 2008.

G. Bastianini-D.N. Sedley, 9. Commentarium in Platonis «Theaetetum», in CPF III: Commentari, Firenze 1995, pp. 227-562.

H.I. BeLL, Bibliography: Graeco-Roman Egypt. A. Papyri (1920-1921), JEA 8, 1922, pp. 83-101.

L. BELlONI, 'Ipponatte' poeta vagante (Callim. Ia. I, fr. 191 Pf.), in Poesia e religione in Grecia. Studi in onore di G. Aurelio Privitera, a c. di M. Cannatà Fera-S. Grandolini, I, Napoli 2000, pp. 71-80.

Poetae lyrici Graeci, ed. T. BergK, II, Poetae elegiaci et iambographi, Lipsiae $1882^{4}\left(1866^{3} ; 1853^{2} ; 1843^{1}\right)$.

L. BERGSON, Kallimachos, Iambos I (fr. 191 Pf.), 26-28, Eranos 84, 1986, pp. 11-16.

B. BERRUECOS FRANK, Heródoto ó $\mu \eta \imath \iota \omega ́ \tau \alpha \tau$ coc. La transformación del léxico homérico en las Historias de Heródoto, Nova Tellus 32, 2015, pp. 115-171.

F. BLASS, Vermischtes zu den griechischen Lyrikern und aus Papyri, RhM 55, 1900, pp. 91-103.

F. BLASS, Die neuen Fragmente griechischer Epoden, RhM 55, 1900, pp. 341-347.

F. BLASS, Literarische Texte mit Ausschluss der christlichen, APF 3, 1906, pp. 257-299.

K. BLouin, Papyri in Paris: The Greek Papyrus Collection in 
BOEDEKER 2002

\section{BOEHLAU 1901}

BossI 1976

Bossi 1978-1979a

Bossi 1978-1979b

BossI $1990^{2}$

BossI-Tosi 1979-1980

BOUDON-MiLLOT 2010

BOWMAN-COLES-GONISOBBINK-PARSONS 2007

BOWRA 1933

BRASWELL 2013

BRAVO 2012

BREMMER 1983

BROGGIATO 2001

BROWN 1984

BROWN 1997

BÜHLER 1964

BÜLOW-JACOBSEN 1982

BURZACCHINI $1977^{1}\left(2005^{2}\right)$ the Bibliothèque Nationale de France, in "Proceedings of the 27th International Congress of Papyrology", Ed. by T. Derda-A. Łaitar-J. Urbanik, Warsaw 2016, II, pp. 853-881. D. BOEDEKER, Epic Heritage and Mythical Patterns in Herodotus, in E.G. Bakker-I.J.F. de Jong-H. van Wees (eds.), Brill's Companion to Herodotus, Leiden-Boston 2002, pp. 97-116.

J. BoeHlau, Ein neuer Erosmythus, Philologus 60, 1901, pp. 321-329.

F. BossI, Nota a Hippon. fr. 78,12 W., QUCC 22, 1976, pp. 2728.

F. Bossi, Archil. fr. 47 W., MCr 13-14, 1978-1979, pp. 41-43.

F. BossI, Hippon. fr. 79,16 W., MCr 13-14, 1978-1979, pp. 149151.

F. BossI, Studi su Archiloco, Bari 1990² (Bologna 1984¹).

F. BossI-R. TosI, Strutture lessicografiche greche, BIFG 5, 19791980, pp. 7-20.

V. Boudon-Millot, L'ecdotique des textes médicaux grecs et l'apport des traductions orientales, in Entre Orient et Occident: la philosophie et la science gréco-romaines dans le monde arabe (Entretiens sur l'antiquité classique 57), Vandœuvres-Genève 2010, pp. 321-364.

Oxyrhynchus: a City and its Texts, Ed. by A.K. BowMAN-R.A. Coles-N. Gonis-D. ObBink-P.J. Parsons, London 2007.

C.M. BowrA, Early lyric and elegiac poetry, in J.U. POWELL, New Chapters in the History of Greek Literature, III, Oxford 1933, pp. 1-67.

Didymos of Alexandria: Commentary on Pindar, Ed. by B.K. BRASWELL, Basel 2013.

B. BRAVo, Per la storia del testo di Erodoto e di quello di Tucidide nell'antichità. Parte prima: le testimonianze dei papiri, Eos 99, 2012, pp. 23-65.

J.N. BREMMER, Scapegoat rituals in Ancient Greece, HSCP 87, 1983, pp. 299-320.

Cratete di Mallo: I frammenti, ed., introd. e note a c. di M. Broggiato, La Spezia 2001.

C.G. Brown, Ruined by Lust: Anacreon, fr. 44 Gentili (432 PMG), CQ 34, 1984, pp. 37-42.

C.G. Brown, Iambos, in D.E. Gerber, A Companion to the Greek Lyric Poets, Leiden-New York-Köln 1997, pp. 11-88.

W. BüHLER, Archilochos und Kallimachos, in Archiloque (Entretiens sur l'antiquité classique 10), Vandœuvres-Genève 1964, pp. 223-253.

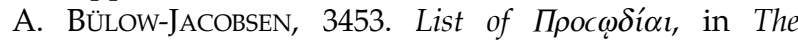
Oxyrhynchus Papyri, XLIX, ed. with Transl. and Notes by A. BÜLOW-JACOBSEN-J.E.G. Whitehorne, London 1982, pp. 95-97.

G. BURZACCHINI, Lirica monodica e Lirica corale, in Lirici greci. Antologia, a c. di E. Degani-G. BurZACCHINI, Firenze $1977^{1}$ [rist. con aggiorn. bibl. a c. di M. Magnani, Bologna 2005²], pp. 121-348. 
BURZACCHINI 2003

BURZACCHINI 2010a

BURZACCHINI 2010b

CAG

CALDERINI 1921

CALDERINI 1942

CANTARella 1944

CANTORE 2013

CAPASSO-HICKEY 2007

CAREy 2009

CARLINI 1986

CARLINI 1999

CASANOVA 1989

CASSIO 1993

CatPErc

Cavallo 1975

Cavallo 1983

Cavallo 1984

CAVAllo 1986

Cavallo 1991
G. BuRZACCHINI, Spunti serio-comici nella lirica greca arcaica, in Incontri triestini di filologia classica (I 2001-2002), Trieste 2003, pp. 191-257.

G. BURZACCHINI, Un recupero editoriale: l'ultimo Ipponatte di Enzo Degani, in Incontri triestini di filologia classica (VIII 2008-2009), Trieste 2010, p. VII (Abstract) e pp. 1-22.

G. BuRZACCHINI, Rec. a AdRADOs 2010", Eikasmós 21, 2010, pp. 591-594.

Commentaria in Aristotelem Graeca.

A. CALDERINI, Testi recentemente pubblicati. 3, Aegyptus 2, 1921, pp. 79-80.

A. CALDERINI, Testi recentemente pubblicati, Aegyptus 22, 1942, p. 130.

R. CANTARella, Gli epodi di Strasburgo, Aegyptus 24, 1944, pp. 1-112.

R. CANTORE, Per la storia del testo di Erodoto. Studi sulla famiglia romana, Bologna 2013.

M. CAPASSO-T. HiCKEY, Un frammento delle Storie di Erodoto (I 59,6-60,1; I 60,4) in PCair JE 45623, SEP 4, 2007, pp. 7376.

C. CAREY, Iambos, in The Cambridge Companion to Greek Lyric, Ed. by F. Budelmann, Cambridge 2009, pp. 149-167.

A. CARLINI (a c. di), Papiri letterari greci della Bayerische Staatsbibliothek di Monaco di Baviera, Stuttgart 1986.

A. CARLINI, Eryxias, in CPF I.1 ${ }^{* * *}$, pp. 485-486.

A. CASANOVA, Eroda 8.5 e le ore della notte, Prometheus 15, 1989, pp. 135-142.

A.C. CASSIO, Parlate locali, dialetti delle stirpi e fonti letterarie nei grammatici greci, in Dialectologica Graeca, "Actas del II Coloquio Internacional de Dialectología Griega" (Miraflores de la Sierra [Madrid], 19-21 de junio de 1991), ed. E. Crespo-J. L. García Ramón-A. Striano, Madrid 1993, pp. 73-90.

Catalogo dei Papiri Ercolanesi, sotto la direzione di Marcello Gigante, Napoli 1979.

G. CAVALLO, $\Gamma \rho \alpha^{\prime} \mu \mu \alpha \tau \alpha$ 'A $\lambda \varepsilon \xi \alpha v \delta \rho \hat{\imath} v \alpha$, JÖByz 24, 1975, pp. 23-54 (= CAVALLO 2005, pp. 175-202).

G. CAVALLO, Libri scritture e scribi a Ercolano, (Suppl. a CronErc 13), Napoli 1983.

G. CAVALLO, I rotoli di Ercolano come prodotti scritti: quattro riflessioni, S\&C 8, 1984, pp. 5-30.

G. CAVAllo, Conservazione e perdita dei testi greci: fattori materiali, sociali, culturali, in Società romana e impero tardoantico, IV, Tradizione dei classici. Trasformazioni della cultura, a c. di A. Giardina, Roma-Bari 1986, pp. 83-172, 246-271 (rist. in G. Cavallo, Dalla parte del libro. Storie di trasmissione dei classici, Urbino 2002, pp. 49-175).

G. CAVAllo, La scrittura greca libraria tra i secoli I a.C.-I d.C. Materiali, tipologie, momenti, in Paleografia e codicologia greca, 
Cavallo 2005

Cavallo 2008

Cavallo-Maehler 1987

Cavallo-Maehler 2008

CAVINI 1985

CAVIni-Donnini Macciò-

FUNGHI-MANETTI 1985

Chambers-Cockle-

TURNER-PARSONS 1981

Chantraine 1968-1980

CIAMPI 2007

CITTI 2015

CMG

COHN 1895

COHN 1898

Collart 1939

Colomo-Del Corso 2013

ColonNa 1955

COMPARETTI 1908

Coppola 1928

Coppola 1929

$\mathrm{CPF}$ in "Atti del II Colloquio internazionale" (Berlino-Wolfenbüttel, 17-21 ottobre 1983), a c. di D. Harlfinger-G. Prato, con la coll. di M. D'Agostino-A. Doda, Alessandria 1991, pp. 11-29 (= CAVALLO 2005, pp. 107-122).

G. CAVALLO, Il calamo e il papiro. La scrittura greca dall'età ellenistica ai primi secoli di Bisanzio, Firenze 2005.

G. CAVALLO, La scrittura greca e latina dei papiri. Una introduzione, Pisa-Roma 2008.

G. CaVallo-H. MaeHLer, Greek Bookhands of the Early Byzantine Period, A.D. 300-800, London 1987.

G. CAVAllo-H. MaeHLer, Hellenistic Bookhands, Berlin-New York 2008.

W. CAVINI, La negazione di frase nella logica greca, in CAVINIDONNINI MACCIÒ-FunGHI-MANETTI 1985, pp. 7-126.

W. CAVInI-M.C. DonNini Macciò-M.S. FunGHI-D. MANETTI, Studi su papiri greci di logica e medicina (STCPF 1), Firenze 1985.

M. Chambers-W.E.H. CoCKLE-E. TuRner-P.J. PARsons (eds.), P.Oxy. 3372-3383. Herodotus, in The Oxyrhynchus Papyri XLVIII, London 1981, pp. 22-29.

P. Chantraine, Dictionnaire étymologique de la langue grecque. Histoire des mots, I-IV, Paris 1968-1980.

A. CIAMPI, Euforione: testo e scolî in PSI XIV 1390, in Comunicazioni dell'Istituto Papirologico «G. Vitelli» 7, Firenze 2007, pp. 9-28.

F. CiтTI, Serse e Demarato (ben. 6, 31, 1-10): Seneca, Erodoto e le declamazioni di argomento storico, SIFC, IV s., 13, 2015, pp. 232-249.

Corpus Medicorum Graecorum

L. CoHN, s.v. Apollonios (83), in RE II, Stuttgart 1895, col. 140.

L. CoHn, Der Attizist Philemon, Philologus 57, 1898, pp. 353-367.

P. COllart, Les papyrus de l'Iliade et de l'Odyssée, RPh 65, 1939, pp. 289-307.

D. Colomo-L. Del CoRso, Un'annotazione problematica (in margine a P.Oxy. XVIII 2181: Platone, Fedone), APapyrol 25, 2013, pp. 55-63.

A. Colonna, Rec. a Pfeiffer 1949-1953, Paideia 10, 1955, pp. $45-47$.

Papiri Greco-Egizi, volume secondo. Papiri fiorentini (fascicolo primo), per cura di D. ComparetTI, Milano 1908.

G. Coppola, Un nuovo frammento dei Giambi di Ipponatte, RFIC n.s. 6, 1928, pp. 500-506.

1089. Nuovo frammento di Ipponatte, in Papiri greci e latini (Pubblicazioni della Società Italiana), IX, Firenze 1929, p. 136.

Corpus dei Papiri filosofici greci e latini, Firenze 1989- ; CPF IV.2 (2002) = Corpus dei papiri filosofici greci e latini Parte IV.2, Tavole (I.1 e III), 2002; CPF IV.2 (2008) = Corpus dei pa- 
CPF I.1.2 (1992)

CRIBIORE 1996

CRIBIORE 2001

CRÖNERT 1901

CRÖNERT 1903

CRÖNERT 1908

CRUSIUS 1906

Cunningham 1971

D' Alessio 2007

DALE 1957

DARIS 1997

De FALCO 1923

DE RicCI 1902

DEBORD 1995

DEGANI 1970-1972

DEGANI $1977^{1}\left(2005^{2}\right)$

DEGANI 1978-1979

DEGANI 1980

DEGANI 1980-1982

DEGANI 1981a

Degani 1981b

DEGANI $1984^{1}\left(2002^{2}\right)$ piri filosofici greci e latini Parte IV.2, Tavole (I.2 Galenus-Isocrates), 2008.

Hermippus 4T?, in Corpus dei papiri filosofici greci e latini (CPF). Testi e lessico nei papiri di cultura greca e latina, I Autori noti, 1**, Firenze 1992, p. 267.

R. CRIBIORE, Writing, Teachers and Students in Graeco-Roman Egypt, Atlanta (GA) 1996.

R. CRIBIORE, Gymnastics of the Mind. Greek Education in Hellenistic and Roman Egypt, Princeton 2001.

W. CRÖNERT, Litterarische Texte mit Ausschluss der christlichen, APF 1, 1901, pp. 104-120; 502-539.

W. CRÖNERT, Litterarische Texte mit Ausschluss der christlichen, APF 2, 1903, pp. 337-381.

W. CRÖNERT, Rec. a P.Flor. II, LZB 59, 1908, pp. 1201-1202.

O. CRUsius, Zur Charakteristik der antiken Jambographen, SBAW 3, 1906, pp. 378-381.

Herodas. Mimiambi, Ed. with Introd., Comm. and Appendices by I.C. CunNINGHAM, Oxford 1971.

G.B. D'AlEssIO, Note su PSI XIV 1391, in Comunicazioni dell'Istituto Papirologico «G. Vitelli» 7, Firenze 2007, pp. 75-80.

A.M. DALE, New material, Lustrum 2, 1957, pp. 6-8.

S. DARIS, Rec. a CPF III, Aegyptus 77, 1997, pp. 147-149.

V. De FAlCo, L'Epicureo Demetrio Lacone, Napoli 1923.

S. DE RICCI, Bulletin papyrologique, REG 15, 1902, pp. 408460.

P. DeBord, Les routes royales en Asie Mineure Occidentale, in P. Briant (éd.), Dans les pas des Dix-Mille. Peuples et pays du Proche-Orient vus par un Grec, "Actes de la Table Ronde Internationale", Toulouse 3-4 février 1995, Toulouse 1995, pp. 89-97.

E. DeGANI, Note al primo epodo di Strasburgo, MCr 5/7, 19701972, pp. 63-80 [rist. in DEGANI 2004, pp. 5-22].

E. DeGANI, Giambo e Elegia, in Lirici greci. Antologia, a c. di E. DEGANI-G. Burzacchini, Firenze $1977^{1}$ [rist. con aggiorn. bibl. a c. di M. Magnani, Bologna 2005²], pp. 1-120.

E. DeGANI, Per un'edizione critica di Ipponatte (I), MCr 13-14, 1978-1979, pp. 153-168.

E. Degani, Rec. a West 1974, Gnomon 52, 1980, pp. 512516 [rist. in DEGANI 2004, pp. 80-84]

E. DeGANI, Hipponactea, MCr 15-17, 1980-1982, pp. 47-50.

E. DEGANI, Considerazioni su tradizione testuale diretta e indiretta nei giambografi greci, in La critica testuale greco-latina, oggi. Metodi e problemi, "Atti del Convegno internazionale (Napoli 29-31 ottobre 1979)”, a c. di E. Flores, Roma 1981, pp. 289-298 [rist. in DEGANI 2004, pp. 85-94].

E. DEGANI, Ipponatte nella letteratura latina, RFIC 109, 1981, pp. 385-406.

E. DEGANI, Studi su Ipponatte, Bari $1984^{1}$ [nuova ed. anastaticamente riprodotta Hildesheim-Zürich-New York 2002²]. 
DEGANI $1991^{2}\left(1983^{1}\right)$

DEGANI 1993

Degani 1995

Degani 1998

DEGANI 2004

DEGANI 2007

DEGNI 1998

DEGNI 2002

DEICHGRÄBER 1930

DEICHGRÄBER 1933

Del CORSO 2006

Del FABBro 1979

Del Grande 1942

Del GRANDE 1948

Del Mastro 2010

DEPEW 1992

DETTORI 2000

Di Benedetto 1984

Di GREGORIO 1975
Hipponax. Testimonia et Fragmenta, ed. H. DEGANI, Stutgardiae-Lipsiae $1991^{2}$ (Leipzig 1983²).

E. DEGANI, Aristofane e la tradizione dell'invettiva personale in Grecia, in Aristophane (Entretiens sur 1'Antiquité classique 38), Vandœuvres-Genève 1993, pp. 1-49 [rist. in DEGANI 2004, pp. 414-462].

E. Degani, Ipponatte e i poeti filologi, Aevum(ant) 7, 1995, pp. 105-136 [rist. in DEGANI 2004, pp. 131-162].

E. DeGanI, Marginalia Hipponactea, Eikasmós 9, 1998, pp. 11-15 [rist. in DEGANI 2004, pp. 163-167].

Filologia e storia. Scritti di Enzo Degani, I-II, a c. di M.G. Albiani-G. Alvoni-A. Barbieri-F. Bossi-G. Burzacchini-F. Citti-F. Condello-E. Esposito-A. Lorenzoni-M. Magnani, O. Montanari-S. Nannini-C. Neri-V. Tammaro-R. Tosi, Hildesheim-Zürich-New York 2004.

Ipponatte. Frammenti, introd., trad. e note di E. DEGANI, premessa di G. Burzacchini, aggiornamenti di A. Nicolosi, Bologna 2007.

P. DegNI, 58. P.Flor. II 115, in Scrivere libri e documenti nel mondo antico, a c. di G. Cavallo-E. Crisci-G. Messeri-R. Pintaudi (Papyrologica Florentina XXX), Firenze 1998, pp. 138-139.

P. DegnI, in Papiri letterari della Biblioteca Medicea Laurenziana, CD ROM, Cassino 2002.

K. DEICHGRÄBER, Die griechische Empirikerschule. Sammlung der Fragmente und Darstellung der Lehre, Berlin-Zürich 1965 (1930).

K. DeICHGRÄBER, Die ärztliche Standesethik des hippokratischen Eides, QGMed 3 (1933), pp. 79-99 (ora in Antike Medizin, a c. di H. Flashar, Darmstadt 1971, pp. 94-120).

L. Del Corso, Lo 'stile severo' nei P.Oxy.: una lista, Aegyptus 86, 2006, pp. 81-106.

M. Del FABBro, Il commentario nella tradizione papiracea, StudPap 18, 1979, pp. 69-132.

C. Del GRANDE, Intorno agli epodi di Strasburgo, in C. DeL GRANDE, Note filologiche, Napoli 1942, pp. 9-36.

C. Del Grande, Ancora sull'età di composizione del I epodo di Strasburgo ([Arch.] fr. 79 D.), GIF 1, 1948, pp. 255-257.

G. Del MASTRO, Il Catalogo descrittivo dei Papiri Ercolanesi, CErc 40, 2010, pp. 215-222.

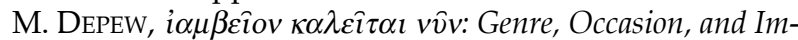
itation in Callimachus, frr. 191 and 203 Pf., TAPhA 122, 1992, pp. 313-330.

Filita grammatico. Testimonianze e frammenti, introd., ed. e comm. a c. di E. DeTTORI, Roma 2000.

V. Di Benedetto, Democrito in Pap. Flor. 115, PP 39, 1984, pp. $41-43$.

L. Di Gregorio, Scholia vetera in Hesiodi Theogoniam, Milan 1975. 
Di GREGORIO 2004

Di MARIA 1996

DICKEY 2007

DiCKS 1970

DIEHL $1917^{3}$

DIEHL 1937

DIEHL $1952^{3}$

DIETZ 1834

DIK 1995

DINDORF 1855

DORANDI 1995

DOVER 1960

DUFFY 1983

DunBAR 1995

DuNN 1988

DÜRING 1957

DYCK 1991

EDMONDS 1931

EDWARDS 1929
Eronda. Mimiambi (V-XIII), a c. di L. Di Gregorio, Milano 2004.

G. Di MARIA, Achillis quae feruntur Astronomica et in Aratum opuscula: De universo, De Arati vita, De Phaenomenorum interpretatione, Palermo 1996.

E. DiCKeY, Ancient Greek Scholarship. A Guide to Finding, Reading, and Understanding Scholia, Commentaries, Lexica, and Grammatical Treatises, from their Beginnings to the Byzantine Period, New York 2007.

D.R. DicKs, Early Greek Astronomy to Aristotle, Ithaca (New York) 1970.

E. DIEHL, Supplementum Lyricum. Neue Bruchstücke von Archilochus, Alcaeus, Sappho, Corinna, Pindar, Bacchylides, Bonn $1917^{3}$ (Supplementum Lyricum. Neue Bruchstücke von Archilochus, Alcaeus, Sappho, Corinna, Pindar, Bonn $1908^{1}$ [1910²]). E. DiEHL, Hypomnema: de Callimachi librorum fatis capita selecta, Acta Universitatis Latviensis, IV 2, Riga 1937, pp. 305-476.

Anthologia lyrica Graeca, ed. E. DIEHL, I/3 Iamborum scriptores, cur. R. Beutler, Lipsiae $1952^{3}\left(1936^{2} ; 1925^{1}\right)$.

F.R. DIETZ, Scholia in Hippocratem et Galenum, Amsterdam, Hakkert 1966 [rist. di Königsberg 1834].

H. DIK, Word Order in Ancient Greek. A Pragmatic Account of Word Order Variation in Herodotus, Amsterdam 1995.

Xenophontis Expeditio Cyri, ed. L. DINDORF, Oxford $1855^{2}$.

T. DORANDI, Un papiro ercolanese a Leiden, ZPE 109, 1995, pp. 35-38.

K.J. Dover, Greek Word Order, Cambridge 1960.

J.M. DufFY, Byzantine Medicine in the Sixth and Seventh Centuries, in J. Scarborough (ed.), Symposium on Byzantine Medicine (Dumbarton Oaks Papers 38), Washington 1983, pp. 21-27.

Aristophanes. Birds, Ed. with Introd. and Comm. by N. DUNBAR, Oxford 1995.

G. DunN, Syntactic Word Order in Herodotean Greek, Glotta 66, 1988, pp. 63-79.

I. DÜRING, Aristotle in the Ancient Biographical Tradition, Göteborg 1957.

A.R. DYCK, The Fragments of Alexander of Cotiaeum, ICS 16, 1991, pp. 307-335.

Greek Elegy and Iambus. Being the Remains of All the Greek Elegiac and Iambic Poets from Callinus to Crates with the Anacreontea, Ed. and Transl. by J.M. EDMONDS, II, Cambridge (Mass.)-London 1931.

W.M. EDWARDS, $\Delta \imath \alpha \lambda o \gamma o c, \delta \imath \alpha \tau \rho \imath \beta \eta, \mu \varepsilon \lambda \varepsilon \tau \eta$, in New Chapters in the History of Greek Literature, Second Series, Some Recent Discoveries in Greek Poetry and Prose Chiefly of the Fourth Century B.C. and Later Times, Ed. by J.U. Powell-E.A. Barber, Oxford 1929, pp. 88-124. 
ERBSE 1960

ERBSE 1961

ERBSE 1971

ERLER 1993

EsPOSITO 2005

EsPOSITO 2009a

ESPOSITO 2009b

EsPOSITO 2012²

ESPOSITO 2014

FABRINI-MANETTI 2008

FAKAS 2001

FALIVENE 1993

FARAGGIANA DI SARZANA 1978 C. FARAGGIANA DI SARZANA, Il commentario procliano alle Opere e i giorni I, Aevum 52, 1978, pp. 17-40

FARINA 1963

FAUSTI 1986

FERRARIO 2000

FEYERABEND 1973

FEYERABEND 1976

FLACH 1877

FLACH 1876

H. ERBSE, Beiträge zur Überlieferung der Iliasscholien, München 1960.

H. ERBSE, Überlieferungsgeschichte der griechischen klassischen und hellenistischen Literatur, in $\mathrm{H}$. Hunger et al., Geschichte der Textüberlieferung der antiken und mittelalterlichen Literatur, I: Antikes und mittelalterliches Buch- und Schriftwesen. Überlieferungsgeschichte der antiken Literatur, Zürich 1961, pp. 207-307.

Scholia Graeca in Homeri Iliadem (scholia vetera), volumen secundum scholia ad libros E-I continens, rec. H. ERBSE, Berlin 1971.

M. ERLER, Philologia medicans, in W. Kullmann-J. Althoff (edd.), Vermittlung und Tradierung des Wissen in der griechischen Kultur, Tübingen 1993, pp. 281-303.

E. EsPosito, P.Oxy. XVII 2087 e una citazione dal ПEPI $\triangle I K A-$ IOCYNHC di Aristotele, ZPE 154, 2005, pp. 79-85.

E. EsPosito, Fragments of Greek Lexicography in the Papyri, TC 1/2, 2009, pp. 255-297.

E. Esposito, 3. Frammenti di commentario, in Comunicazioni dell'Istituto Papirologico «G. Vitelli» 8, Firenze 2009, pp. 1016.

E. EsPosito, Aristophanes 2, in CLGP I.1.4, Berlin-Boston 2012², pp. 250-252.

E. EsPOSITO, O.Berol. inv. 12605 riconsiderato: testo, traduzione e commento, Eikasmós 25, 2014, pp. 159-175.

P. FABRINI-D. MANETtI, 1. Aphorismi I 1-3 (cum titulo): PAnt 28, in CPF I.2, Firenze 2008, pp. 77-82.

C. FAKAS, Der hellenistische Hesiod: Arats Phainomena und die Tradition der antiken Lehrepik, Wiesbaden 2001.

M.R. Falivene, Callimaco serio-comico: il primo Giambo (fr. 191 Pf.), in Tradizione e innovazione nella cultura greca da Omero all'età ellenistica. Scritti in onore di B. Gentili, III, a c. di R Pretagostini, Roma 1993, pp. 911-925.

A. FARINA, Ipponatte, introd., testo crit., testim., trad., comm. con appendice e lessico, Napoli 1963.

D. FAUSTI, P. München 24, in CARLINI 1986, pp. 25-30.

M. FERRARIO, La nascita della filologia epicurea: Demetrio Lacone e Filodemo, CronErc 30, 2000, pp. 53-61.

B. Feyerabend, Hippokrates Aphorismoi II 14-24, ZPE 10, 1973, pp. 122-128.

19. Hippokrates, Aphorismoi 2, 14-24, in Kölner Papyri, bearb. von B. Kramer-R. Hübner, Opladen 1976, pp. 54-56. J. FLACH, De fontibus grammaticis scholiorum ad Hesiodi Opera et Dies, Jahrbücher für classische Philologie 23, 1877, pp. 433-440.

J. FLACH, Glossen und Scholien zur hesiodischen Theogonie mit Prolegomena, Leipzig 1876. 
FLEISCHER 1969

FOWLER 1979

FOWLER 1984

FOWLER 1987

FRACCAROLI 1910

FRAENKEL 1942

FRAENKEL 1957

FRÄNKEL $1969^{3}$

FRASER 1972

FUCHS 1927

FÜHRER 2013

FUNGHI-

MesSeri SAVORELLI 1992

GAISFORD 1823

GALIANO 1958

GALLAZZI 1981

GALLAZZI-LEHNUS 2001

GALLI 1938

GALLi 1940

GAROFALO 2003

GÄRTNER 2008
U. FleISCHER, Rec. a P.Ant. III, Gnomon 41, 1969, pp. 640-641. L.M. FowLER, Reconstructing the Cologne Alcaeus, ZPE 33, 1979, pp. 17-28.

D. FOWLER, Sceptics and Epicureans, OSAPh 2, 1984, pp. 237267.

R.L. FowLER, The Nature of Early Greek Lyric: Three Preliminary Studies, Toronto-Buffalo-London 1987.

Lirici greci, tradotti da G. FRACCAROLI, I Elegia e giambo, Torino 1910.

E. Fraenkel, An Epodic Poem of Hipponax, CQ 36, 1942, pp. 54-56.

E. FRAENKEL, Horace, Oxford 1957, pp. 24-36 [trad. it. Orazio, a c. di S. Lilla, Roma 1993, pp. 36-52].

H. FrÄNKEL, Dichtung und Philosophie des frühen Griechentums. Eine Geschichte der griechischen Epik, Lyrik und Prosa bis zur Mitte des fünften Jahrhunderts, München $1969^{3}$ (1962²; 19511ㅣㄹ. [trad. it. Poesia e filosofia nella Grecia arcaica. Epica, lirica e prosa greca da Omero alla metà del V secolo, Bologna 1997]. P.M. Fraser, Ptolemaic Alexandria, I-III, Oxford 1972.

R. FuchS, Rec. a NACHMANSON 1925, BPhW 47, 1927, pp. 545-548.

R. FÜHRER, Zur Archilochos-Epode BKT X 11, ZPE 185, 2013, p. 60.

M.S. FunGHI-G. MESSERI SAVORELLI, Note papirologiche e paleografiche, Tyche 7, 1992, pp. 75-88.

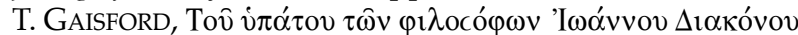

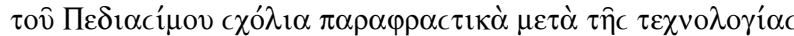

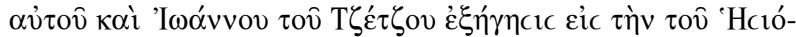
$\delta o v$ 'Ac $\pi i \delta \alpha$, in Poetae Minores Graeci II, Leipzig 1823, pp. 609-654.

M. Fernández-GALIANO, La lírica griega a la luz de los descubrimientos papirológicos, in "Actas del Primer Congreso español de Estudios clásicos" (Madrid, 15-19.4.1956), Madrid 1958, pp. 59-180.

C. GallazZI, P.Mil.Vogl. inv. 1196: Homerus, Ilias VII, 412424, ZPE 42, 1981, pp. 45-46.

C. GALLAZZI-L. LeHNus, Due nuovi frammenti delle Diegeseis, ZPE 137, 2001, pp. 7-18.

U. GALLI, Note agli epodi di Strasburgo, A\&R 6, 1938, pp. 157175.

U. Galli, Postille agli epodi di Strasburgo, A\&R 8, 1940, pp. 255-267.

I. GAROFAlO, I sommari degli Alessandrini, in Galenismo e medicina tardoantica. Fonti greche, latine e arabe, "Atti del Seminario Internazionale di Siena", Certosa di Pontignano - 9 e 10 settembre 2002, a c. di I. Garofalo-A. Roselli, Napoli 2003, pp. 203-231.

T. GÄRTNER, Kritische Bemerkungen zu den Fragmenten des Hipponax, WS 121, 2008, pp. 53-66. 
GeMELli Marciano 1996

GENTILI 1958

GEORGACAS 1984

GERBER 1970

GERBER 1999

GERCKE 1900

GigANTE 1990

GMP

GOMME-ANDREWES 1970

GONIS 2009

GOUREVITCH 1984

GOUREVITCH 1988

GRENFELL 1919

GRENFELL-GOODSPEED

GRENFELL-Hunt 1898

GRENFELL-Hunt 1901

GRENFELL-Hunt 1903

GRENFELL-HunT 1908

GRENSEMANN 1965
M.L. Gemelli Marciano, Rec. a CPF III, MH 53, 1996, p. 306.

Anacreon, ed. B. GeNTILI, Roma 1958.

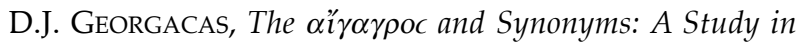
Greek Etymology, in Studies Presented to Sterling Dow in his Eightieth Birthday, Ed. by A.L. Boegehold-W.M. Calder IIIJ. McK. Camp II-W.T. Loomis-K.J. Rigsby-L. Threatte-S.V. Tracy-J.S. Traill, Durham (N.C.) 1984, pp. 101-120.

D.E. GERBER, Euterpe. An Anthology of Early Greek Lyric, Elegiac, and Iambic Poetry, Amsterdam 1970.

Greek Iambic Poetry, Ed. and Transl. by D.E. Gerber, Cambridge (Mass.)-London 1999.

A. GERCKE, Zwei neue Fragmente der Epoden des Archilochos, WKPh 17, 1900, pp. 28-30.

M. Gigante, Quelques précisions sur le scepticisme et l'Épicurisme, in Le scepticisme antique. Perspectives historiques et systematiques, "Actes du Colloque international sur le scepticisme antique", Lausanne 1-3 juin 1988 (Cahiers de la revue de théologie et de philosophie 15), Lausanne 1990, pp. 68-93.

Greek Medical Papyri, I, a c. di I. Andorlini, Firenze 2001.

A.W. Gomme-A. Andrewes-K.J. Dover, A Historical Commentary on Thucydides, IV: Books V 25-VII, Oxford 1970.

N. GonIS, Abbreviations and Symbols, in The Oxford Handbook of Papyrology, Ed. by R.S. Bagnall, Oxford 2009, pp. 170-178.

D. GOUREVITCH, Le triangle hippocratique dans le monde grécoromain (Bibliothèque des Écoles françaises d'Athènes et de Rome 251), Rome 1984.

D. Gourevitch, Rec. a CAVINI-Donnini Macciò-FunghIMANETTI 1985, RHS 41.2, 1988, pp. 216-217.

1619. Herodotus iii, in The Oxythynchus Papyri, XIII, Ed. with Trans. and Notes by B.P. GRENFELL-A.S. Hunt, London 1919, pp. 180-189.

B.P. GRenfell-E.J. Goodspeed, The Tebtunis Payri, II, London 1907.

19. Herodotus I. 76, in The Oxyrhynchus Papyri, I, Ed. with Trans. and Notes by B.P. GrenfELl-A.S. HunT, London 1898, pp. 45-46.

P.Amh. II 12. Aristarchus' Commentary on Herodotus, in The Amherst Papyri, I, by B.P. Grenfell-A.S. Hunt, London 1901, pp. 3-4.

The Oxyrhynchus Papyri, III, Ed. with Trans. and Notes by B.P. GRENFELL-A.S. HUNT, London 1903.

853. Commentary on Thucydides II, in The Oxyrhynchus Papyri, VI, Ed. with Trans. and Notes by B.P. GRENFELL-A.S. HuNT, London 1908, pp. 107-149.

H. GRENSEMANN, Rec. a AleXANDERSON 1963, Gymnasium 72, 1965, pp. 261-263. 
GRONEWALD 1978

GRONEWALD 1981

GUARDASOLE 1997

HADOT 1987

HANKINSON 1988

HANSON 1985

HANSON 1987

HANSON 1990

HANSON 1992

HANSON 1995

HANSON 1997a

HANSON 1997b

HANSON 1998

HANSON 2001

HANSON 2005

HANSON 2008

HANSON 2016

HANSON-GAGOS 1997
M. GRONEWALD, Einige Fackelmann-Papyri, ZPE 28, 1978, pp. 271-277.

M. GRONEWALD, Glossar mit Zitaten aus Herodot und Telekleides, ZPE 42, 1981, pp. 8-10.

Eraclide di Taranto. Frammenti, a c. di A. GuARDASOlE, Napoli 1997.

I. HADOT, Les introductions aux commentaires exégétiques chez les auteurs platoniciens et les auteurs chrétiens, in Les règles de l'interprétation, ed. par L. Tardieu, Paris 1987, pp. 99-122.

R. HANKInson, Rec. a CAVINI-DONNINI Macciò-FunGHIMANETTI 1985, BHM 62, 1988, pp. 296-297.

A.E. Hanson, Papyri of Medical Content, YClS 28, 1985, pp. 25-47.

A.E. Hanson, Rec. a Cavini-Donnini Macciò-FunGHIMANETTI 1985, HPLS 9, 1987, pp. 348-350.

A.E. Hanson, Rec. a MANETTI 1985, Society for Ancient Medicine \& Pharmacy Newsletter 18, 1990, p. 54.

A.E, HANSON, Rec. a MANETti 1992, Society for Ancient Medicine Newsletter 20, 1992, pp. 135-136.

A.E. HANsON, Well Articulated Spaces: Hippocrates, Epidemics II.6.7-22 and Aphorisms II 14-24, Society for Ancient Medicine Review 23, 1995, pp. 26-27.

A.E. HANSON, Fragmentation and the Greek Medical Writers, in G.W. Most (ed.), Collecting Fragments / Fragmente sammeln, Göttingen 1997 (Aporemata 1), pp. 289-314.

A.E. HANSON, Rec. a BKT IX, SAMR 24, 1997, p. 162.

A.E. HANSON, In the Shadow of Galen: Two Berlin Papyri of Medical Content, in Text and Tradition. Studies in Ancient Medicine and its Transmission Presented to Jutta Kollesch, Ed. by K.-D. Fischer-D. Nickel-P. Potter, Leiden-Boston-Köln 1998, pp. 145-159.

A.E. HANSON, A Quotation from Galen Nat. fac. (BKT IX 81), in Greek Medical Papyri, a c. di I. Andorlini, Firenze 2001, pp. 101-106.

A.E. Hanson, Greek Medical Papyri from the Fayum Village of Tebtunis: Patient Involvement in a Local Health-care System?, in Hippocrates in Context, "Papers read at the XIth International Hippocrates Colloquium", University of Newcastle upon Tyne 27-31 August 2002, Ed. by Ph.J. van der Eijk (Studies in Ancient Medicine 31), Leiden-Boston 2005, pp. 387-402.

A.E. HANSON, Galenus 4T, in CPF I.2, Firenze 2008, pp. 4850 .

A.E. HANSON, The Hippocratic Aphorisms in Ptolemaic and Roman Egypt, in L.D. Jones-R.M. Rosen, Ancient Concepts of the Hippocratic, "Papers Presented at the XIIIth International Hippocratic Colloquium", Austin, Texts, August 2008, Leiden-Boston 2016, pp. 48-60.

A.E. Hanson-T. Gagos, Well Articulated Spaces: Hippocrates 
HaSLAM 1978

HASLAM 1980

HASLAM 1991

HaSLAM 1992

HASLAM 1994

HASlAM 1995

HASLAM 1997

HASLAM 1998

HasLam 2011

HaSLAm 2013

HATZIDAKIS 1923

HATZILAMBROU 2002

Hauvette 1901

Hauvette 1905

HEADLAM-KNOX 1922

VAN HeEl 1989

HeIBERg 1927

HEMMERDINGER 1981

HERSHBELl 1993
Epidemics II 6, 7-22, in Specimina per il Corpus dei Papiri Greci di Medicina, a c. di I. Andorlini, Firenze 1997, pp. 117-140. M. Haslam, Apollonius Rhodius and the Papyri, ICS 3, 1978, pp. 47-73.

3329. Lexicon (Diogenianus?), in The Oxyrhynchus Papyri, XLVII, Ed. with Transl. and Notes by R.A. Coles, M.W. HASLAM, London 1980, pp. 44-48.

M. Haslam, On Ancient Manuscripts of the "Republic", Mnemosyne 4a s., 44, 1991, pp. 336-346.

M.W. HaSlam, Homeric Readings Lost and Found, CPh 87, 1992, pp. 322-325.

M.W. Haslam, The Homer Lexicon of Apollonius Sophista: I. Composition and Constituents; II. Identity and Transmission, CPh 89, 1994, pp. 1-45; 107-119.

4100-4112. Thucydides, in The Oxyrhynchus Papyri, LXI, Wd. with Trans. and Notes by T. Gagos-M.W. HASLAM-N. Lewis, London 1995, pp. 59-60.

M. Haslam, Homeric Papyri and Transmission of the Text, in A New Companion to Homer, Ed. by I. Morris-B. Powell, Leiden-New York 1997, pp. 55-100.

4455. Commentary on Herodotus, Bk. 5, in The Oxyrhynchus Papyri, LXV, Ed. with Trans. and Notes by M. HASLAM-A. Jones-F. Maltomini-M.L. West, London 1998, pp. 55-59.

M.W. Haslam, Apollonius Rhodius, in Commentaria et Lexica Graeca in Papyris reperta (CLGP), edd. G. Bastianini-M. Haslam-H. Maehler-F. Montanari-C. Römer, adiuvante M. Stroppa, pars I Commentaria et lexica in auctores, vol. 1 Aeschines-Bacchylides, fasc. 3 Apollonius Rhodius-Aristides, Berlin 2011, pp. 3-42.

M. Haslam, Attestations of $\dot{\eta}$ Kovvr in BKT 10.4 (Hom. Od. 15.531-553 with Marginal Annotations), BASP 50, 2013, pp. 203-206.

G.N. HatZIDAKIS, Neugriechisches, Glotta 12, 1923, pp. 148150.

R.G. Hatzilambrou, A Duke Papyrus of Herodotus IV. 144.2145.1, BASP 39, 2002, 41-45.

A. HAuvetTE, Les nouveaux fragments d'Archiloque, REG 14, 1901, pp. 71-91.

A. Hauvette, Archiloque, Paris 1905.

Herodas. The Mimes and Fragments, with Notes by W. HEADLAM, Ed. by A.D. KNOX, Cambridge $1922\left(1966^{2}\right)$ [rist. London 2001].

J. vAN HEEL, Un frammento perduto del PHerc. 831, BCPE 19, 1989, pp. 187-191.

J.L. HEIBERG, Hippocratis opera, CMG I 1 (1927).

B. HeMmerdinger, Les manuscrits d'Hérodote et la critique verbale, Genova 1981.

J.P. Hershbell, Plutarch and Herodotus - the Beetle in the Rose, RhM 136, 1993, 143-163. 
HERTER 1973

HIRSCHBERGER 2004

HOLFORD-STREVENS-

VARDI 2004

HOMBERT 1939

HOMBERT 1946-1947

HOMBERT 1951

HORAK 1992

HORNBLOWER 2006

HORNBLOWER 2013

HOUSTON 2009

HoUSTON 2014

How-WeLls $1928^{2}$

Hude 1927

HulTSCH 1882

HUMPERS 1921

HuNT 1910

HunT 1911

HunT 1911

HuNT 1914

HunT 1927

HunT 1938

HUNTER 2009
H. Herter, Kallimachos aus Kyrene, RE Suppl. 13, 1973, coll. 184-266.

M. Hirschberger, Gynaikon Katalogos und Megalai Ehoiai: Ein Kommentar zu den Fragmenten zweier hesiodeischer Epen, München-Leipzig 2004.

The worlds of Aulus Gellius, Ed. by L. HOLFORD-STREVEnsA. VARDI, Oxford 2004.

M. HOMBeRT, Rec. a P.Ryl. III, CE 14, 1939, pp. 174-178.

M. HOMBert, Bulletin Papyrologique XX, REG 49-50, 19461947, pp. 381 e 388.

M. Hombert, Rec. a MASSON 1950, CE 51, 1951, p. 172.

U. HORAK, Illuminierte Papyri Pergamente und Papiere, I, Wien 1992.

S. Hornblower, Herodotus' Influence in Antiquity, in The Cambridge Companion to Herodotus, Ed. by C. Dewald-J. Marincola, Cambridge-New York 2006, pp. 306-318.

Herodotus, Histories, Book V, Ed. S. Hornblower, Cambridge 2013.

G.W. Houston, Papyrological Evidence for Book Collections and Libraries in the Roman Empire, in Ancient Literacies. The Culture of Reading in Greece and Rome, Ed. by W.A. JohnsonH.N. Parker, Oxford 2009, pp. 233-267.

G.W. Houston, Inside Roman Libraries: Book Collections and Their Management in Antiquity, Chapel Hill 2014.

W.W. How-J. Wells, A Commentary on Herodotus with Introduction and Appendixes, Oxford $1928^{2}$.

Herodoti Historiae, ed. X. HuDE, Oxford $1927^{3}$.

F. Hultsch, Griechische und Römische Metrologie, Berlin $1882^{2}$.

A. HuMPERs, Gloses homériques sur ostrakon, RPh 45, 1921, pp. 90-92.

1012. Treatise on Literary Composition, in The Oxyrhynchus Papyri, VII, Ed. with Transl. and Notes by A.S. HunT, London 1910, pp. 82-103.

1092. Herodotus ii, VII, in The Oxyrhynchus Papyri, VIII, Ed. with Transl. and Notes by A.S. HuNT, London 1911, pp. 127-135.

Catalogue of the Greek and Latin Papyri in the John Rylands Library, Manchester, Vol. I, Ed. by A.S. HunT, London 1911. 1233. Alcaeus, in The Oxyrhynchus Papyri, X, Ed. with Transl. and Notes by B.P. Grenfell-A.S. HuNT, London 1914, pp. 50-70.

The Oxyrhynchus Papyri, Ed. with Transl. and Notes by A.S. HuNT, XVII, London 1927.

The Tebtunis Papyri, III 2, Ed. by A.S. HunT-J.G. SmylyC.C. Edgar, London 1938.

R. HunTER, Hesiod's Style: Towards an Ancient Analysis, in Brill's Companion to Hesiod, Ed. by F. Montanari-A. Rengakos-Chr. Tsagalis, Leiden-Boston 2009, pp. 253-269. 
HUNTER 2011

HUNTER 2014

IERACI BIO 1993

IERACI BIO 1999

IERACI BIO 2003

IERACI BIO 2006

IHM 2002

INDELLI 2008

IRIGOIN 1975 (2003)

IRIGOIN 1977

IRIGOIN 1994

ISAGER 1998

ISKANDAR 1976

JACHMANN 1949

JACOBY 1913

JACOBY 1930

JACOBY 1954

JANNI 1984
R. Hunter-D. Russell, edd., Plutarch: How to Study Poetry (De audiendis poetis), Cambridge-New York 2011.

R. Hunter, Hesiodic Voices: Studies in the Ancient Reception of Hesiod's Works and Days, Cambridge-New York 2014.

A.M. IERACI BIO, I papiri medici bizantini, MAT 17, 1993, pp. 3-51.

A.M. IERACI BIO, Tracce della fortuna di terapie ippocratiche in età bizantina, in I. Garofalo-A, Lami-D. Manetti-A. Roselli, Aspetti della terapia nel Corpus Hippocraticum, "Atti del IX Colloque International Hippocratique", Pisa 25-29 settembre 1996, Firenze 1999, pp. 455-465.

A.M. IERACI BIO, Disiecta membra della scuola iatrosofistica alessandrina, in Galenismo e medicina tardoantica. Fonti greche, latine e arabe, "Atti del Seminario Internazionale di Siena", Certosa di Pontignano - 9 e 10 settembre 2002, a c. di I. Garofalo-A. Roselli, Napoli 2003, pp. 9-51.

A.M. IERACI BIO, Un inedito commento anonimo ad Ippocrate (Aph. I 1), in Ecdotica e ricezione dei testi medici greci, "Atti del V Convegno Internazionale" (Napoli, 1-2 ottobre 2004) a c. di V. Boudon-Millot et al., Napoli 2004, pp. 253-271.

S. IHM, Clavis commentariorum der antiken medizinischen Texte, Leiden 2002.

G. INDELLI, Rec. a CPF, Parte I.2 e Parte IV.2 Tavole, Firenze 2008, RFIC 136, 2008, pp. 469-484.

J. IRIGOIN, Tradition manuscrite et histoire du texte. Quelques problèmes relatifs à la Collection hippocratique, in La Collection hippocratique et son rôle dans l'histoire de la médecine, "Colloque de Strasbourg" (23-27 octobre 1972), Leiden, Brill 1975, pp. 3-18 (rist. in La tradition des textes grecs, Paris 2003, pp. 251-269).

J. IRIGOIN, Quelques réflexions sur le concept d'archétype, RHT 7, 1977, pp. 235-245.

J. IRIGOIN, Les éditions de textes, in MONTANARI 1994, pp. 3982; Discussion, pp. 83-93.

S. IsAGER, The Pride of Halikarnassos. Editio Princeps of an Inscription from Salmakis, ZPE 123, 1998, pp. 1-23.

A.Z. ISKANDAR, An Attempted Reconstruction of the Late Medical Curriculum, Medical History 20, 1976, pp. 235-258.

G. JaCHMANN, Vom frühalexandrinischen Homertext, Göttingen 1949.

F. JАСОвY, Herodotos, in RE Suppl. II, Stuttgart 1913, coll. 205-520 (= ID., Griechische Historiker, Suttgart 1956, pp. 7164).

F. JACOBY, Hesiodi Carmina. I, Theogonia, Berlin 1930.

F. JACOBY, FGrHist IIIb (Supplement). A Commentary on the Ancient Historians of Athens, I: Text; II: Notes, Addenda, Corrigenda, Index, Leiden 1954.

P. JANNI, La mappa e il periplo. Cartografia antica e spazio odologico, Roma 1984. 
JOHNSON 1994

JOHNSON 2004

JOHNSON 2009

JOLY-BYL 2003

JOUANNA-MANETTI 2001

JOUANNA 1971

JOUANNA 1975

JOUANNA 2012

JOUANNA 2013

JURENKA 1900

KAKRIDIS 2000

KALLÓs 1951-1952

KASSEL 1958

KASSEL-Austin 1989

KASSEL-Austin 1995

KAZANSKAYA 2014

KEANEY-LAMBERTON 1996

KENYON 1899

KENYON $1951^{2}$

KERKHECKER 1999

KeYSER 1986

KIRKWOOD 1961
W.A. JoHnson, The Function of the Paragraphus in Greek Literary Prose Texts, ZPE 100, 1994, pp. 65-68.

W.A. JoHnson, Bookrolls and Scribes in Oxyrhynchus, Toronto-Buffalo-London 2004.

W.A. Johnson, The Ancient Book, in The Oxford Handbook of Papyrology, Ed. by R.S. Bagnall, Oxford 2009, pp. 256-281. R. Joly-S. BYL, Hippocrate. Du régime, CMG I 2.4, Berlin $2003^{2}$.

J. Jounnna-D. Manetti, A Text Concerning Health and Disease, in GMP I, pp. 85-93.

J. JouANNA, Un nouveau témoignage sur la Collection hippocratique: P. gr. inv. 26, col. III, de Strasbourg, ZPE 8, 1971, pp. 147-60.

J. JouAnNA, Hippocrate, La nature de l'homme, CMG I 1.3, Berlin $1975\left(2002^{2}\right)$.

J. JouAnNA, À la recherche du Pronostic perdu, BAGB 2012, pp. 55-74.

Hippocrate, Pronostic, ed. J. JouAnNA, Paris 2013.

H. JURENKA, Archilochos von Paros, Wien 1906.

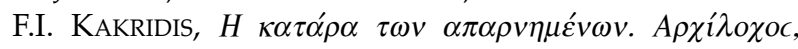

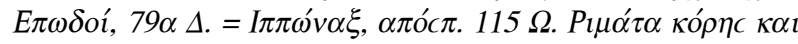

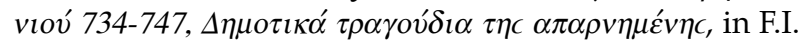
Kakridis-G.M. Sifakis-I. Touloumakos-O. Tsagarakis, $K \tau \varepsilon$ -

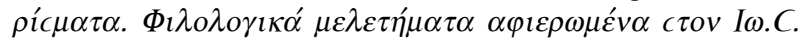

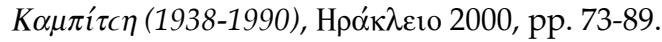

E. KALLÓs, Gloses pour Archiloque, AAntHung 1, 1951-1952, pp. 67-74.

R. KASSEL, Kleinigkeiten zu den Kallimachos-fragmenten, RhM 101, 1958, pp. 235-238.

Poetae comici graeci (PCG), edd. R. Kassel-C. Austin, VII (Menecrates-Xenophon), Berolini-Novi Eboraci 1989.

Poetae comici Graeci (PCG), edd. R. KASSEL-C. Austin, VIII Adespota, Berolini-Novi Eboraci 1995.

M. KAZANSKAYA, Les expressions homériques dans les Histoires d'Hérodote, Lalies 34, 2014, pp. 161-172.

[Plutarch]: Essay on the Life and Poetry of Homere, Ed. by J.J. KeANEY-R. LAMBERTON, Atlanta 1996.

F.G. KENYON, Abbreviations and Symbols, in The Palaeography of Greek Papyri, Oxford 1899, pp. 153-156 [rist. in Abbreviations in Greek Inscriptions, Papyri, Manuscripts and Early Printed Book, Compiled by A.N. Oikonomides, Chicago 1974, pp. 127-130].

F.G. KENYON, Books and Readers in Ancient Greece and Rome, Oxford 1951².

A. KERKHECKER, Callimachus' Book of Iambi, Oxford 1999.

P. KEYSER, Errors of Calculation in Herodotus, CJ 81, 1986, pp. 230-242.

G.M. KIRKWOOD, The Authorship of the Strasbourg Epodes, TAPhA 92, 1961, pp. 267-282. 
KIVILO 2010

KLINGER 1948-1949

KNOx 1929

KoENEN 1977

KolLesch 1978

KONING 2010

KÖRTE 1890

KÖRTE 1913

KÖRTE 1924

KÖRTE 1935

KÖRTE 1941

Kroll 1912

KRÜGER 1990

KuDLIEN 1970

KÜHN-FLEISCHER 1989

LAMA 1991

LAMBERTI 1984

LAMBERTI-MANETTI 2008

LAMI 2008

LAMI-MANETTI 2008

LASSERRE 1950

LASSERRE 1951

LASSERRE 1958
M. KIVILO, Early Greek Poets' Lives: The Shaping of the Tradition, Leiden-Boston 2010.

V. KLINGER, De Archilochi fragmento papyraceo 79 D. ${ }^{2}$ eiusque exordio nondum recognito, Eos 43, 1948-1949, pp. 40-47.

Theophrastus. Characters, Ed. and Transl. by J.M. Edmonds; Herodes. Cercidas and the Greek Choliambic Poets, Ed. and Transl. by A.D. KNOX, Cambridge (Mass.)-London 1929.

L. Koenen, Horaz, Catull und Hipponax, ZPE 26, 1977, pp. 73-93.

J. KOLLESCH, Literarische Texte mit Ausschluss der christlichen, APF 26, 1978, pp. 141-148.

H.H. KonING, Hesiod: The Other Poet (Mnemosyne Supplements vol. 325), Leiden-Boston 2010.

A. KÖRTE, Metrodori Epicurei Fragmenta, JCPh Suppl. 17 (1890), pp. 531-597.

A. KÖRTE, Literarische Texte mit Ausschluss der christlichen, APF 6, 1913, pp. 223-268.

A. KÖRTE, Literarische Texte mit Ausschluss der christlichen, APF 7, 1924, pp. 224-258.

A. KÖRTE, Literarische Texte mit Ausscluss der christlichen, APF 11, 1935, pp. 220-283.

A. KÖRTE, Literarische Texte mit Ausscluss der christlichen, APF 14, 1941, pp. 103-150.

W. KrolL, s.v. Heron (4), in RE VIII, Stuttgart 1912, col. 992. J. KRÜGER, Oxyrhynchos in der Kaiserzeit. Studien zur Topographie und Literaturrezeption, Frankfurt a.M.-Bern-New York-Paris 1990.

F. KudLIEN, Medical Education in Classical Antiquity, in C.D. O'MALLEY, The History of Medical Education, Berkeley-Los Angeles 1970, pp. 3-37.

J.-H. KüHN-U. FLeISCHER, Index Hippocraticus, Göttingen 1989.

M. Lama, Aspetti di tecnica libraria ad Ossirinco: copie letterarie su rotoli documentari, Aegyptus 71, 1991, pp. 55-120.

P. LAMBERTI, Rec. a MARGANNE 1981, HPLS 6, 1984, pp. 110112.

Hippocrates 3 (P.Köln 19), Hippocrates 9 (P.Ant. 184), Hippocrates 12 (P.Ant. 184), in CPF I 2, Firenze 2008, pp. 85-88; 113-125, 130-133.

Hippocrates 13, De victu in acutis (App.) 24-27 (PRyl 56), in CPF I.2, Firenze 2008, pp. 134-137.

A. Lami-D. ManetTI, De articulis 8, P. Stras. Inv. G 26, in CPF I.2, Firenze 200, pp. 198-201.

F. LASSERre, Les Épodes d'Archiloque, Paris 1950.

F. LASSERRE, Rec. a SCHUbART 1950, AC 20, 1951, pp. 187188.

Archiloque. Fragments, texte établi par F. LASSERRE, trad. et comm. par A. BONNARD, Paris 1958. 
LATTE 1929

LATTE 1948

LAVAGNINI 1929

LEGRAND 1942

LEHMANN 1880

LEHRS $1882^{3}$

LEITH 2007

LEITH 2009

LEITH 2014

LEITH 2017

LEITH (in stampa)

LENAERTS 1996

LENAERTS 2000

LEO 1900

LGGA

LIEBER 1981
K. LATTE, Hipponacteum, Hermes 64, 1929, pp. 385-388 [rist. in Kleine Schriften zu Religion, Recht, Literatur und Sprache der Griechen und Römer, München 1968, pp. 464467].

K. LATTE, De nonnullis papyris Oxyrrhynchiis. I. De Hipponactis Epodo, Philologus 97, 1948, pp. 37-47 [rist. in Kleine Schriften zu Religion, Recht, Literatur und Sprache der Griechen und Römer, München 1968, pp. 468-477].

B. LaVAGNinI, Sul nuovo frammento dei giambi d'Ipponatte, Ann. Univ. Toscane n.s. 12, 1929, pp. 3-15 [rist. in Da Mimnermo a Callimaco, Torino 1950, pp. 56-66; ATAKTA. Scritti minori di filologia classica bizantina e neogreca, Palermo 1978, pp. 225-237].

PH.-E. LegRAND, Hérodote. Introduction. Notice préliminaire su la vie et la personnalité d'Hérodote et sur la présente édition, Paris 1942.

O. LEHMANN, Die tachygraphischen Abkürzungen der griechischen Handschriften, Leipzig 1880 [rist. Hildesheim 1965].

K. LeHRS, De Aristarchi studiis Homericis, Lipsiae $1882^{3}$.

D. LeITH, The Hippocratic Oath in Antiquity and on Papyrus, in Zwischen Magie und Wissenschaft. Ärzte und Heilkunst in den Papyren aus Ägypten, hrsg. von H. Froschauer-C. Römer, Wien 2007, pp. 35-42.

The Oxyrhynchus Papyri, LXXIV, Ed. with Transl. and notes by D. LEITH-D.C. Parker-S.R. Pickering-N. GonisM. Malouta, London 2009.

The Oxyrhynchus Papyri, LXXX, Ed. with Transl. and Notes by M. HiRT-D. LeITH-W.B. Henry, London 2014.

D. LEITH, The Hippocratic Oath in Roman Oxyrhynchus, in En marge du Serment hippocratique: contrats et serments dans le monde gréco-romain, "Actes de la Journée d'étude internationale" (Université de Liège, 29 octobre 2014), par M.-H. Marganne-A. Ricciardetto (Papyrologica Leodiensia 7), Liège 2017, pp. 39-50.

D. LEITH, Asclepiades of Bithynia as Hippocratic Commentator, in corso di stampa in "Proceedings of the XVth Colloque Hippocratique" (Manchester 28-30 October 2015).

J. LENAERTS, Rec. a CPF III, CE 71, 1996, pp. 352-354.

J. LENAERTS, Rec. a P.Oxy. LXV, CE 150, 2000, pp. 348-352.

F. LEO, De Horatio et Archilocho, (Diss.) Gottingae 1900 [rist. in Ausgewählte kleine Schriften, II Zur römischen Literatur der Kaiserzeit. Zur griechischen Literatur. Rede zur Säcularfeier Karl Lachmanns, hrsg. und eingel. von E. Fraenkel, Roma 1960, pp. 139-157].

F. Montanari-F. Montana-L. Pagani, Lexicon of Greek Grammarians of Antiquity, Brill Online, http: / / referenceworks. brillonline.com/browse/lexicon-of-greek-grammariansof-antiquity.

E. LIEBER, Galen in Hebrew: the Transmission of Galen's Works 
LiESHOUT 1980

LINGUITI 1987

LINNÉR 1941

LiUZZO 2014

LIVREA 2003

LLOYD 1989

LOBEL 1941a

LOBEL 1941b

LOBEL 1948

LOBEL 1951

LOBEL 1954

LOBEL 1962

LOBEL 1964

LOBEL 1971

LOBEL 1977

LÖFFLER 1999

LONGO 1996

LORIMER 1947

LUENZNER 1866

LUISELLI 2011

LUISELLI-MANETTI 2008 in the Mediaeval Islamic World, in Galen: Problems and Prospects, Ed. by V. Nutton, London 1981, pp. 167-185.

R.G.A. van LIESHOUT, Greeks on Dreams, Utrecht 1980.

A. Linguiti, Rec. a CAVINI-Donnini Macciò-FungHIMANETTI 1985, Elenchos 8, 1987, p. 491.

S. LinNéR, Herodotea. II. De ordine verborum in papyris servato, Eranos 39, 1941, pp. 147-148.

P.M. LiUzzo, Frammenti di Erodoto. Problemi e metodi nello studio della storiografia frammentaria, Tesi di Dottorato, Bologna 2014.

E. LivreA, Callimachi Iambus V, ZPE 144, 2003, pp. 51-58. Erodoto. Le storie, II: Libro II. L'Egitto, Introd., testo e comm. a c. di A.B. Lloyd, Trad. di A. FrASChetTI, Milano 1989. 2174-5. Hipponax,'I $\alpha \mu \beta$ ol, in The Oxyrhynchus Papyri, XVIII, Ed. with Transl. and Notes by E. LOBEL-C.H. Roberts-E.P. Wegener, London 1941, pp. 67-87.

2176. Commentary on Hipponax, in The Oxyrhynchus Papyri, XVIII, Ed. with Transl. and Notes by E. LOBEL-C.H. RobertsE.P. Wegener, London 1941, pp. 87-96 e pp. 184-185.

Additions and Corrections to Pieces Published in Previous Volumes, in The Oxyrhynchus Papyri, XIX, Ed. with Transl. and Notes by E. LOBEL-E.P. Wegener-C.H. Roberts-H.I. Bell, London 1948, pp. 147-154.

2297. Alcaeus, in The Oxyrhynchus Papyri, XXI, Ed. with Notes by E. LoBEL, London 1951, pp. 49-59.

The Oxyrhynchus Papyri, XXII, Ed. with Transl. and Notes by E. LOBEL-C.H. Roberts, London 1954.

The Oxyrhynchus Papyri, XXVIII, Ed. with Notes by E. LOBEL, London 1962.

The Oxyrhynchus Papyri, XXX, Ed. with Notes by E. LOBEL, London 1964.

The Oxyrhynchus Papyri, XXXVII, Ed. with Notes by E. LOBEL, London 1971.

3213. Lyric Verses in 'Doric', in The Oxyrhynchus Papyri, XLV, Ed. with Notes by A.K. Bowman-M.W. Haslam-S.A. Stephens-M.L. West, London 1977, pp. 14-18.

D. LÖFfleR, Zu Hipponax Fragment 78,14 Degani $(78,14$ West), Prometheus 25, 1999, pp. 35-36.

A. LoNGO, Rec. a CPF III Commentari, Firenze 1995, RFIC 124, 1996, pp. 199-206.

H.L. LORIMER, The Hoplite Phalanx with Special Reference to the Poems of Archilochus and Tyrtaeus, The Annual of the British School at Athens 42, 1947, pp. 76-138.

E. LUENZNER, Epaphroditi grammatici quae supersunt (diss. Bonn 1866).

R. Luiselli, Aratus, in Commentaria et Lexica Graeca in Papyris reperta (CLGP) I.1.3, Berlin-Boston 2011, pp. 43-155.

R. LuiselLi-D. MANETTI, Hippocrates 24T (PRyl 530), in CPF I.2, Firenze 2008, pp. 180-197. 
XXXIV

LUNDON 1997

LUNDON-MATthaIOS 2005

LUPI 2014

LUPI 2017

LUPPE 1973

LUPPE 1975a

LUPPE 1975b

LUPPE 1980

LuPPE 1990

LUPPE 1992

LUPPE 2001

LUSCHNAT 1954

LUZZATTO 2012

MAAS 1934

MAAS 1942

MAASS 1892

MAASS 1898

MAEHLER 1986

MAgdelaine 1994

MAgdeLAine 1996
Conspectus librorum

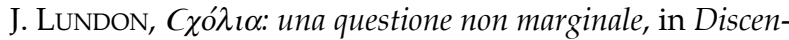
tibus obvius. Omaggio degli allievi a Domenico Magnino, Como 1997, pp. 73-86.

J. LunDON-S. MATTHAIOS, Nominal Accidents by Question and Answer: Two Fragments of a Té $\chi v \eta \Gamma \rho \alpha \mu \mu \alpha \tau \imath \kappa \eta$, One New, ZPE 154, 2005, pp. 97-116.

S. LuPI, Essere uomini, diventare donne: $i$ Lidi di Coricio di Gaza tra storia e riflessione sui meccanismi di costruzione dell'identità individuale, in Alta formazione e ricerca in Sardegna, "Atti del convegno di studi 'Giovani ricercatori' " (Sassari, 16 dicembre 2011), a c. di E. Cicu-A Gavini-M. Sechi, Raleigh (NC) 2014, pp. 135-149.

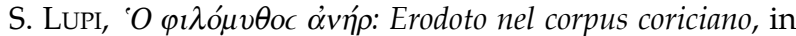
L'Ecole de Gaza: espace littérarie et identité culturelle dans l'Antiquité tardive, "Actes du Colloque International de Paris", College de France, 23-25 Mai 2013, éd. par E. Amato-A. Corcella-D. Lauritzen, Leuven-Paris-Bristol (CT) 2017, pp. 267-286.

W. Luppe, Rec. a Lobel 1971, Gnomon 45, 1973, pp. 321330.

W. LUPPE, Rec. a WeST 1974, DLZ 96, 1975, pp. 689-692.

W. LupPE, Rec. a Austin 1973, GGA 227, 1975, pp. 179-206.

W. LuPPE, Literarische Texte unter Ausschluss der christlichen, APF 78, 1980, pp. 233-250.

W. Luppe, Zum Hipponax-Kommentar P.Oxy. 2176 (fr. 1 Kol. II, fr. 129c Degani), Philologus 134, 1990, pp. 155-158.

W. LuPPE, Literarische Texte: Drama, APF 38, 1992, pp. 7586.

W. LuPPE, Rec. a P.Oxy. LXV, Gnomon 73, 2001, pp. 1-5.

O. LusCHNAT, Die Thukydidesscholien. Zu ihrer handschriftlichen Grundlage, Herkunft und Geschichte, Philologus 98, 1954, pp. $14-58$.

M.T. LuZZATTO Commentare Demostene (le strategie dell'hypomnema nel Didimo di Berlino), BollClass, $3^{a}$ s., 32, 2012, pp. 3-72.

P. MAAS, Rec. a NORSA-Vitelli 1934, Gnomon 10, 1934, pp. 436-439.

P. MAAS, Commentarii in Hipponactem P.Oxy. 2176 fragm. 6, CQ 36, 1942, p. 133.

E. MAASS, Aratea, Berlin 1892.

E. MAASS, Commentariorum in Aratum reliquiae, Berlin 1898. H. MAEHLER, Literarische Texte: Lyrik, APF 32, 1986, pp. 81 e 85 .

C. Magdelaine, Histoire du texte et édition critique, traduite et commentée, des Aphorismes d'Hippocrate, Thèse pour le Doctorat Nouveau Régime, Univ. Paris-Sorbonne, Paris IV, 1994.

C. Magdelaine, Le commentaire de Damascius aux Aphorismes d'Hippocrate, in Storia ed ecdotica dei testi medici greci, 
MAGDELAINE 2003

MALITZ 1990

MANETTI 1985

MANETTI 1986

MANETTI 1992

MANETTI 1995a

MANETTI 1995b

MANETTI 1996a

MANETTI 1996b

MANETTI 2007

MANETTI 2008

MANETTI 2014

MANETTI 2015
"Atti del II Convegno Internazionale", Parigi 24-26 maggio 1994, a c. di A. Garzya, Napoli 1996, pp. 289-306.

C. Magdelaine, Le commentaire de Palladius aux Aphorismes $d^{\prime}$ Hippocrate et les citations d'Al-ya'qu - bi-, in Trasmissione e Ecdotica dei testi medici greci, "Atti del IV Convegno Internazionale", Parigi 17-19 maggio 2001, a c. di A. GarzyaJ. Jouanna, Napoli 2003, pp. 321-334.

J. MaLITZ, Das Interesse an der Geschichte. Die griechischen Historiker und ihr Publikum, in Purposes of History. Studies in Greek Historiography from the 4 th to the 2 nd centuries B.C., "Proceedings of the International Colloquium", Leuven, 24-26 May 1988, Ed. by H. Verdin-G. Schepens-E. de Keyser (Studia Hellenistica 30), Leuven 1990, pp. 323-349.

D. MANETTI, Tematica filosofica e scientifica nel papiro fiorentino 115. Un probabile frammento di Galeno In Hippocratis De alimento, in CAVINI-DONNINI MACCIÒ-FUNGHI-MANETTI 1985, pp. 173-206.

D. MANETti, P. München 43, in CARLINI 1986, pp. 97-101.

D. MANETTI, PBerol 1173A e i commenti tardoantichi a Galeno, in Tradizione e ecdotica dei testi medici tardoantichi e bizantini, "Atti del Convegno Internazionale", Anacapri, 29-31 ottobre 1990, a c. di A. Garzya, Napoli 1992, pp. 211-235.

D. MANeTTI, Commentarium in Galeni «De sectis», in CPF III, Firenze 1995, pp. 19-38.

D. MANETTI, 4. Commentarium in Hippocratis «De alimento", in CPF III, Firenze 1995, pp. 39-51.

D. ManetTI, Due frammenti inediti di Berlino del VI/VII ${ }^{p}$ (PBerol inv. 11739B), in ODOI DIZHSIOS. Le vie della ricerca, Studi in onore di Francesco Adorno, a c. di M.S. FunGHI, Firenze 1996, pp. 25-30.

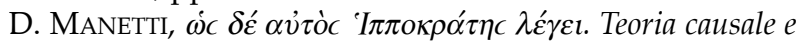
ippocratismo nell'Anonimo Londinese, in Hippokratische Medizin und antike Philosophie, hrsg. von R. Wittern-P. Pellegrin (Medizin der Antike I), Hildesheim 1996, pp. 295-310. D. MANETTI, La cognizione del dolore. Per una interpretazione di P.Oxy. 437, in La science médicale antique. Nouveaux regards, Études réunis en l'honneur de Jacques Jouanna, sous la direction de V. Boudon-Millot - A. Guardasole C. Magdelaine (edd.), Paris 2007, pp. 307-314.

Galenus 6? (P.Flor. 115); Hippocrates 22T (P.Berol. 9764); Hippocrates $23 T$ (P.Ant. 124); Hippocrates $31 T$ (P.Oxy. 437), in CPF I.2, Firenze 2008, pp. 51, 176-178, 178-180, 197-198, 206-207, 207-209.

Alle origini dell'ippocratismo: fra IV e III secolo, in J. JouannaM. Zink, Hippocrate et les hippocratimses: médecine, religion, societé, "XIV e Colloque International Hippocratique", Paris, 2014, pp. 217-251.

Medicine and Exegesis, in Brill's Companion to Ancient Greek 
MANETti-Roselli 1982

MANetTi-Roselli 1994a

MANETti-Roselli 1994b

MANFREDI 1974

MANFREDI 1979

MANFREDI 1986

MANIERI 1998

MANKIN 1995

MANSFELD 1994

MARCH 1987

MARCH 1988

MARGANNE 1981

MARGANNE 1984

MARGANNE 1986a

MARGANNE 1986b

MARGANNE 1996

MARGANNE 1998a

MARGANNE 1998b

MARGANNE 2000

MARGANNE 2004
Scholarship, Ed. by F. Montanari-S. Matthaios-A. Rengakos, Leiden-Boston 2015, pp. 1126-1215.

D. MANETTI-A. Roselli, Ippocrate, Epidemie, libro VI (Biblioteca di Studi Superiori LXVI), Firenze 1982.

D. MANETTI-A. RoselLI, Galeno commentatore di Ippocrate, ANRW II 37.2, 1994, pp. 1529-1635.

D. MANETTI-A. RoselLI, Il ruolo della tradizione nei libri chirurgici di Celso, in La médecine de Celse, Aspects historiques, scientifiques et littéraires, textes réunis et édités par G. Sabbah-Ph. Mudry, Saint-Étienne 1994, 103-112.

M. MANFREDI, PFlor 115, SIFC n.s. 46, 1974, pp. 154-184.

M. MANFREDI, PSI XV, fasc. I, Firenze 1979, pp. 37-39.

V. MANFREDI, La strada dei Diecimila. Topografia e geografia dell'Oriente di Senofonte, Milano 1986.

A. MANIERI, L'immagine poetica nella teoria degli antichi. Phantasia ed enargeia, Pisa-Roma 1998.

Horace. Epodes, Ed. by D. MANKIN, Cambridge 1995.

J. MANSFELD, Prolegomena. Questions to be Settled before the Study of an Author or a Text (Philosophia antiqua 61), Leiden 1994.

J.R. MARCH, The Creative Poet: Studies on the Treatment of Myths in Greek Poetry (BICS Suppl. 49), London 1987.

J.R. MARCH, Peleus and Thetis in the Catalogue of Women, in "Proceedings of the XVIII International Congress of Papyrology", Athens 1988, I, pp. 345-352.

M.-H. MARGANNE, Inventaire analytique des papyrus grecs de médecine, Genève 1981.

M.-H. Marganne, La "collection médicale" d'Antinoopolis, ZPE 56, 1984, pp. 117-126.

M.-H. MarganNe, Rec. a CAVINI-DonNini Macciò-FunGHIMANETTI 1985, AIHS 36, 1986, pp. 179-180.

M.-H. MARGANNE, Compléments à $l$ ' "Inventaire analytique des papyrus grecs de médecine", ZPE 65, 1986, pp. 175-186.

M.-H. Marganne, La médecine dans l'Égypte romaine: les sources et les méthodes, ANRW II 37.2, 1996, pp. 2709-2740. M.-H. MARGANNE, La chirurgie dans l'Égypte gréco-romaine d'après les papyrus littéraires grecs (Studies in Ancient Medicine 17), Leiden-Boston 1998.

M.-H. Marganne, Présence d'Hippocrate dans les papyrus grecs de médecine, in A. Verbanck-Piérard, Au temps d'Hippocrate. Médecine et societé en Grèce antique, Catalogue d'exposition, Musée Royal de Mariemont 1998, pp. 105-113.

M.-H. MARGANNE, Autre version ou commentaire du Régime, II, 49 hippocratique?, in Papyri in honorem Johannis Bingen actuagenari, ed. H. Melaerts (Studia varia Bruxellensia ad orbem graeco-latinum pertinentia V), Leuven 2000, pp. 110.

M.-H. MARGANNE, Le livre médical dans le monde gréco-romain (Cahiers du CEDOPAL 3), Liège 2004. 
MARGANNE 2008

MARINCOLA 2006

MARKOVICH 1978

MARTANO 2002

MARTANO 2004a

MARTANO 2004b

MARTANO 2005

MARTANO 2006

MARTANO 2008

MARTIN 1956

MARTIN 1974

MARZILLO 2010

MASSON 1946-1947

MASSON 1949

MASSON 1950

MAsSON 1951

MASSON 1955

MASSON 1962a

MASSON 1962b

MATIJAŠIĆc 2013
Hippocrates 37 T (P.Bingen 1 = P.Tebt. 897), CPF I.2, Firenze 2008, pp. 228-233.

J. MARINCOLA, Herodotus and the Poetry of the Past, in The Cambridge Companion to Herodotus, Ed. by C. Dewald-J. Marincola, Cambridge-New York 2006, pp. 13-28.

M. MARKOVICH, Eraclito. Frammenti (Biblioteca di Studi Superiori LXIV), Firenze 1978.

A. Martano, Scolii e glosse allo Scudo di Eracle dal manoscritto Ambrosiano C 222 Inf., Aevum 76, 2002, pp. 151-200.

A. MARTANo, Brevi note a P.Oxy. 4652, Aegyptus 84, 2004, pp. 129-136.

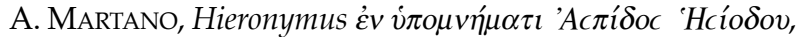
in Lyco of Troas and Hieronymus of Rhodes: Text, Translation, and Discussion, Ed. by W.W. Fortenbaugh-S.A. White, New Brunswick-London 2004, pp. 457-474.

A. MARTANO, La tradizione manoscritta dell'esegesi antica allo Scudo di Eracle esiodeo: la famiglia del Vat. Gr. 1332 (sec. XIIIXV), Aevum 79, 2005, pp. 461-489.

A. MARTANo, L'esegesi antica allo Scudo di Eracle nell'Etymologium Genuinum e Gudianum, in I classici greci e i loro commentatori. Dai papiri ai marginalia rinascimentali, a c. di G. Avezzù-P. Scattolin (Memorie dell'Accademia Roveretana degli Agiati ser. II vol. X), Rovereto 2006, pp. 85-120.

A. MARTANO, La tradizione manoscritta dell'esegesi antica allo Scudo di Eracle esiodeo: due gruppi di codici (sec. XIV-XVI), Aevum 82, 2008, pp. 543-580.

J. Martin, Histoire du texte des Phénomènes d'Aratos, Paris 1956.

J. MARTIN, Scholia in Aratum vetera, Leipzig 1974.

P. MARZILlo, Der Kommentar des Proklos zu Hesiods 'Werken und Tagen': Edition, Übersetzung und Erläuterung der Fragmente (Classica Monacensia 33), Tübingen 2010.

O. MASSON, Les "Épodes de Strasbourg": Archiloque ou Hipponax? Et quelques problèmes relatifs au texte d'Hipponax, REG 59-60, 1946-1947, pp. 8-27.

O. MASSON, Sur un papyrus contenant des fragments d'Hipponax (P.Oxy. XVIII. 2176), REG 62, 1949, pp. 300-319.

O. MASsON, Nouveaux fragments d'Hipponax, PP 5, 1950, pp. 71-76.

O. MASSON, Encore les "Épodes de Strasbourg", REG 64, 1951, pp. 427-442.

O. MASSON, Rec. a K. LATTE, Hesychii Alexandrini Lexicon, I, Hauniae 1953, RPh 29, 1955, pp. 286-290.

O. MAsson, Les fragments du poète Hipponax, éd. critique et comm., Paris 1962.

O. Masson, Recherches sur le vocabulaire d'Hipponax, 1. Un mot d'origine égyptienne, ě $\rho \pi \iota, \mathrm{RPh} 36,1962, \mathrm{pp} .46-50$.

I. MATIJAŠIĆ, Aristarco ed Erodoto in Stefano di Bisanzio, RhM 156, 2013, pp. 217-220. 
XXXVIII

MATIJAŠIĆ 2018

MAtThaios 1999

MAtThaios 2015

MAYSER-SCHMOLL $1970^{2}$

MAZZINI-PALMIERI 1991

MCNAMEE 1977

MCNAMEE 1981

MCNAMEE 1985

MCNAMEE 1992

MCNAMEe 1995

MCNAMEE 2007

MCNAMEE 2010

MCNEAL 1983

MECCARIELLO 2014

Medeiros 1959-1960

MedeIros 1961

Medeiros 1969

MELIADÒ 2006

MeNCI 1984
Conspectus librorum

I. MATIJAŠIĆ, Shaping the Canons of Ancient Greek Historiography. Imitation, Classicism, and Literary Criticism, BerlinBoston 2018.

S. MATTHAIOS, Untersuchungen zur Grammatik Aristarchs. Texte und Interpretation zur Wortartenlehre, Göttingen 1999.

S. MATTHAIOS, Greek Scholarship in the Imperial Era and Late Antiquity, in A Companion to Ancient Greek Scholarship, Ed. by S. MatThaios-F. Montanari-A. Rengakos, Leiden-Boston 2015, pp. 184-296.

E. MAYSER, Grammatik der griechischen papyri aus der Ptolemäerzeit. Mit einschluss der gleichzeitigen ostraka und der in Ägypten verfassten inschriften, Berlin $1970^{2}$ (a c. di $\mathrm{H}$. SCHMOLL).

I. MAZZINI-N. PALMieri, L'école medicale de Ravenne, in Les écoles médicales à Rome, "Actes du 2ème Colloque International sur les textes médicaux latins antiques", Lausanne, Septembre 1986. Edition préparée par Ph. Mudry-J. Pigeaud, Genève 1991, pp. 285-310.

K. McNAmEe, Marginalia and Commentaries in Greek Literary Papyri, Diss. Duke University 1977.

K. McNAmeE, Abbreviations in Greek Literary Papyri and Ostraca (BASP Suppl. 3), Ann Arbor (Mich.) 1981.

K. MCNAMEE, Abbreviations in Greek Literary Papyri and Ostraca: Supplement, with List of Ghost Abbreviations, BASP 22, 1985, pp. 205-225.

K. MCNAMEE, Sigla and Select Marginalia in Greek Literary Papyri, Bruxelles 1992.

K. McNamee, Missing links in the Development of Scholia, GRBS 36, 1995, pp. 399-414.

K. McNamee, Annotations in Greek and Latin Texts from Egypt, New Haven (Conn.) 2007.

K. McNameE, Very Small Scripts, in "Proceedings of the Twenty-Fifth International Congress of Papyrology" (Ann Arbor, July 29-August 4, 2007), Ed. by. T. Gagos, Ann Arbor 2010, pp. 521-540.

R.A. MCNeAL, On Editing Herodotus, AC 52, 1983, pp. 110129.

C. MeCCARIEllo, Le hypotheseis narrative dei drammi euripidei. Testo, contesto, fortuna, Roma 2014.

W. DE SOUSA MedeIROS, O milhafre, a garça e o báratro nos fragmentos de Hipónax, Humanitas 11-12, 1959-1960, pp. 133-144.

W. DE Sousa Medeiros, Hipónax de Éfeso, I Fragmentos dos Iambos, Coimbra 1961.

W. DE SOUSA MEDEIROS, Hipponactea. Subsídios para uma nova edição crítica do iambógrafo de Éfeso, Coimbra 1969.

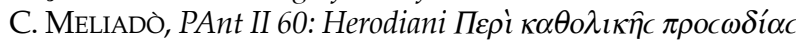
fragmentum novum?, ZPE 155, 2006, pp- 49-54.

G. MENCI, Per l'identificazione di un nuovo stile di scrittura li- 
MERKELBACH 1953

MerKelbaCh-WeST 1967

MerKelbach-West 1990

Mertens-StRauss 1992

MESSERI 1996-1997

MESSERI 2014

MESSERI-PinTAUdi 2002

MetTe 1936

MeYerhof 1930

MiLETTI 2006-2007

MiLeTti 2008

MILLER 1868

MiRALLES 1983

MiRALlEs 2004

MirAlles-PòRTUlAS 1988

MONTANA 2005a

Montana 2005b

MONTANA 2006

MonTANA 2009a

MONTANA 2009b braria greca, in "Atti del XVII Congresso Internazionale di Papirologia", I, Napoli 1984, pp. 51-56.

R. MerKelBACH, Literarische Texte unter Ausschluss der christlichen, APF 15-16, 1953, p. 88.

Fragmenta Hesiodea, ed. R. Merkelbach-M.L. West, Oxford 1967.

Hesiodi fragmenta selecta, ed. R. MERKELBACH-M.L. West, Oxford $1990^{3}$.

P. Mertens-J.A. Strauss, Les papyrus d'Hérodote, ASNP 22, 1992, pp. 969-978.

G. MESSERI, Rec. a CPF III, AnPap 8-9, 1996/97, pp. 259262.

G. MESSERI, Erodoto e Tucidide in due frammenti papiracei fiorentini, in Il miglior fabbro. Studi offerti a Giovanni Polara, a c. di A. De Vivo-R. Perrelli, Amsterdam 2014, pp. 5-16.

G. MESSERI-R. PINTAUDI, I lettori dei papiri: dal commento autonomo agli scoli, in Talking to the Text. Marginalia from Papyri to Print. "Proceedings of a Conference held at Erice", 26 September-3 October 1998, Ed. by V. Fera-G. Ferraù-S. Rizzo, Messina 2002, I, pp. 37-57.

H.J. MetTE, Sphairopoiia: Untersuchungen zur Kosmologie des Krates von Pergamon, Munich 1936.

M. Meyerhof, Von Alexandrien nach Bagdad, Sitzb. Pr. Ak. Wiss., Phil.-hist. Kl., Berlin 1930.

L. MiletTI, I messaggeri del Gran Re in Erodoto. Un'eco eschilea in VIII 98, 2 e le glosse dei commentatori antichi, RAAN 74, 2006-2007, pp. 225-237.

L. MiletTI, Herodotus in Theon's Progymnasmata. The Confutation of Mythical Accounts, MH 65, 2008, pp. 65-76.

E. MiLLER, Mélanges de littérature grecque, contenant un grand nombre de textes inédits, Paris 1868.

C. MiRALLES, Il fr. 78 W. di Ipponatte, QUCC 43 n.s. 14, 1983, pp. 7-16.

C. Miralles, Hipponax's Fragment 78 Dg. (78 W.; $74 \mathrm{Md}$.), in ID., Studies on Elegy and Iambus, Amsterdam 2004, pp. 156-163.

C. Miralles-J. Pòrtulas, Hipponax's Fragment 78 Dg. (78 W.; 74 Md.), in IID., The Poetry of Hipponax, Roma 1988, pp. 9-21.

F. Montana, s.v. Alexander [6] Cotiaeus, in LGGA, 2005.

F. MontanA, s.v. Chaeris, in LGGA, 2005.

F. Montana, s.v. Ammonius [2] Alexandrinus, in LGGA, 2006.

F. Montana, A Hypomnema on Herodotus' bk. 5: Notes to P.Oxy. LXV 4455, Col. I, in Fragments of the Past. Ancient Scholarship and Greek Papyri, Ed. by F. Montanari-S. Perrone, TC 1/2, 2009, pp. 241-254.

F. MONTANA, Storici, filologi, storici-filologi: intersezioni nella cultura ellenistica, in Ingenia Asiatica. Fortuna e tradizione di 
Montana 2011

MONTANA 2012a

MONTANA 2012b

MONTANA 2012c

MonTANA 2012d

MONTANA 2014

MONTANA 2015a

MONTANA 2015b

MONTANA 2015c

MONTANA 2016

MontANA 2018

MONTANARI 1988

MONTANARI 1993

MONTANARI 1994

MONTANARI 1996

MONTANARI 1997a

MONTANARI 1997b

MONTANARI 1997c storici d'Asia Minore, a c. di F. Gazzano-G. Ottone-L. Santi Amantini, Roma 2009, pp. 157-181.

F. MontAnA, The Making of Greek Scholiastic Corpora, in From Scholars to Scholia, Chapters in the History of Ancient Greek Scholarship, Ed. by F. Montanari-L. Pagani, BerlinNew York, pp. 105-161.

F. MontanA, Nuova luce su P.Amh. II 12, Col. I (hypomnema di Aristarco al libro I di Erodoto), ZPE 180, 2012, pp. 72-76.

F. MONTANA, La filologia ellenistica. Lineamenti di una storia culturale, Pavia 2012.

F. MontanA, Ettore, Cebrione e il tethrippon. P.Oxy. 76.5095, fr. $1 \rightarrow$,rr. 10-11, commento a Il. 12.91, APF 58, 2012, pp. 209-215.

F. MontAna, MPER N.S. I 34 + P.Vindob. G 29833 C, in CLGP I $1.4\left(2012^{2}\right)$, pp. 107-113.

F. MontanA, Anything but a Marginal Question. On the

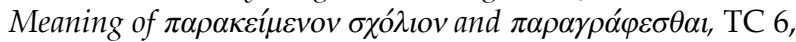
2014, pp. 24-38.

F. MONTANA, Per il testo della redazione A (non alfabetica) delle Lexeis di Erodoto, in Lemmata. Beiträge zum Gedenken an Christos Theodoridis, Ed. by M. Tziatzi-M. Billerbeck-F. Montanari-K. Tsantsanoglou, Berlin-Boston 2015, pp. 431-451.

F. MONTANA, Hellenistic Scholarship, in Brill's Companion to Ancient Greek Scholarship, Ed. by S. Matthaios-F. Montanari-A. Rengakos, Leiden-Boston 2015, pp. 60-183.

F. Montana, s.v. Didymus [2] Claudius, in LGGA, 2015.

F. Montana, Aristarco, Erodoto, l'Egitto: una nota, in "e si d'amici pieno". Omaggio di studiosi italiani a Guido Bastianini per il suo settantesimo compleanno, a c. di A. Casanova-G. Messeri-R. Pintaudi, Firenze 2016, II, pp. 539-546. F. Montana, Alexander of Cotiaeum Teacher, Exegete, Diorthotes, AION(filol) 40, 2018, in stampa.

I frammenti dei grammatici Agathokles, Hellanikos, Ptolemaios Epithetes, ed. F. MonTANARI (SGLG 7), Berlin-New York 1988, pp. 1-128.

F. MONTANARI, L'erudizione, la filologia, la grammatica, in Lo spazio letterario della Grecia antica, direttori G. Cambiano-L. Canfora-D. Lanza, I/2, Roma 1993, pp. 235-281.

La philologie grecque à l'époque hellénistique et romaine, préparés et présidés par F. MONTANARI (Entretiens sur l'Antiquité classique 40), Genève 1994.

F. MONTANARI, s.v. Alexandros [32], in NP 1, Stuttgart 1996, col. 485.

F. Montanari, s.v. Didymos [1] aus Alexandria, in NP 3, Stuttgart 1997, coll. 550-552.

F. Montanari, s.v. Didymus [3] Claudius, in NP 3, Stuttgart 1997, col. 553.

F. MONTANARI, The Fragments of Hellenistic Scholarship, in Collecting Fragments - Fragmente Sammeln, Ed. by G.W. Most, Göttingen 1997, pp. 273-288. 
MONTANARI 2002

MONTANARI 2006

MONTANARI 2009

MONTANARI 2011

MONTANARI 2013

MONTANARI 2015

MONTANARI 2016

MonTANARI-PAGANI 2011

MonTeVeCCHI 1988

MORELLI 1964

MoRelLi 2002

MoRELLI 2010

MoRGAN 1998

MORGENSTERN 1918

Most 2006

Most 2007

MÜLLER 1968
F. MONTANARI, Filologia ed erudizione antica, in Da Aí́v a Eikasmós, "Atti della giornata di studio sulla figura e sull'opera di Enzo Degani (Bologna, 19 aprile 2001)", Bologna 2002, pp. 73-88.

F. MONTANARI, Glossario, parafrasi, 'edizione commentata' nei papiri, in I classici greci e i loro commentatori. Dai papiri ai marginalia rinascimentali, a c. di G. Avezzù-P. Scattolin (Memorie dell' Accademia Roveretana degli Agiati ser. II vol. X), Rovereto 2006, pp. 9-16.

F. MONTANARI, Ancient Scholarship on Hesiod, in Brill's Companion to Hesiod, Ed. by F. Montanari-A. Rengakos-Chr. Tsagalis, Leiden-Boston 2009, pp. 313-342.

F. Montanari, Correcting a Copy, Editing a Text, Alexandrian Ekdosis, and Papyri, in MonTANARI-PAgANi 2011, pp. 1-15.

F. MONTANARI, Gli storici greci e la filologia di età ellenisticoromana, in Le età della trasmissione. Alessandria, Roma, Bisanzio, "Atti delle Giornate di Studio sulla storiografia greca frammentaria", Genova, 29-30 maggio 2012, a c. di F. Gazzano-G. Ottone, Tivoli 2013, pp. 1-32.

F. MONTANARI, Ekdosis. A Product of the Ancient Scholarship, in Brill's Companion to Ancient Greek Scholarship, Ed. by F. Montanari-S. Matthaios-A. Rengakos, Leiden-Boston 2015, pp. 641-672.

F. MONTANARI, Remarks on the Citations of Authors and Works in Ancient Scholarship, TC 8, 2016, pp. 73-82.

From Scholars to Scholia. Chapters in the History of Ancient Greek Scholarship, Ed. by F. MONTANARI-L. PAGANI (TC Supplementary Volumes 9), Berlin 2011.

O. MONTEVECCHI, La papirologia, ristampa riveduta e corretta con Addenda, Milano 1988.

G. Morelli, Due nuove edizioni di Ipponatte, RFIC 92, 1964, pp. 370-376.

G. Morelli, Giambografia, in Da Aiwov a Eikasmós, "Atti della giornata di studio sulla figura e sull'opera di E. Degani (Bologna, 19 aprile 2001)", Bologna 2002, pp. 15-30.

F. Morelli, P.Ant. III 139 fr. 1: un frammento di Galeno, De compositione medicamentorum per genera VII 10 - e di P.Ant. III 186?, ZPE 172, 2010, pp. 203-207.

T.J. MORGAN, Literate Education in the Hellenistic and Roman Worlds, Cambridge 1998.

O. Morgenstern, Sitzungsberichte des Philologischen Vereins zu Berlin 1918, Sokrates 6, 1918, pp. 360-366.

Hesiod I: Theogony, Works and Days, Testimonia, Ed. and Transl. by G.M. Most, Cambridge-London 2006.

Hesiod II: The Shield, Catalogue of Women, Other Fragments, Ed. and Transl. by G.M. Most, Cambridge-London 2007.

W. MüLlER, Griechische literarische Texte auf Papyrus und 
MÜLLER 1974

MuRRAY 1972

MUŠČININA 1987

NACHMANSON 1925

NACHMANSON 1933

NAGY 2009

NAUCK 1848

NERI 1995

NICKAU 1977

NiCOLAI 1992

NicOLAI 2007

NiCOLAI 2008

NicOLOSI 2005

NiCOLOSI 2007

NiCOLOSI 2009

NiCOLOSI 2010

NicOLOSI 2011

NiCOLOSI 2012

NiCOLOSI 2014
Pergament, Nr. 3: Kommentar zu Hesiod, Erga?, Forschungen und Berichte 10, 1968, p. 119.

W. MÜLLER, Bruchstücke untergegangener griechischer Literatur, in Festschrift zum 150 jährigen Bestehen des Berliner Ägyptischen Museums, hrsg. von W. Müller, Berlin 1974, pp. 395-407.

O. Murray, Herodotus and Hellenistic Culture, CQ n.s. 22, 1972, pp. 200-213.

L.N. MuščInINA, Strasburgskie epody i problema ih atribucii, PhilClass 3, 1987, pp. 112-120.

E. NACHMANSON, Ein neuplatonischer Galenkommentar auf Papyrus, Göteborgs Högskolas Arsskrifter 31.2, 1925, pp. 204-208.

E. NACHMANSON, Zum Nachleben der Aphorismen, QGMed 3, 1933, pp. 93-97.

G. NAGY, Hesiod and the Ancient Biographical Traditions, in Brill's Companion to Hesiod, Ed. by in F. Montanari-A. Rengakos-Chr. Tsagalis, Leiden-Boston 2009, pp. 271-311.

Aristophanis Byzantii grammatici Alexandrini fragmenta, collegit et disposuit A. NAUCK, Halle 1848.

C. NeRI, L'empietà del naso (Hippon. fr. 129a,1 Dg.), Eikasmós 6, 1995, pp. 11-14.

K. NICKAU, Untersuchungen zur textkritischen Methode des Zenodotos von Ephesos, Berlin-New York 1977.

R. NiCOLAI, La storiografia nell'educazione antica, Pisa 1992. R. NICOLAI, Storia e storiografia nella scuola greca, in J.A. Fernández-Delgado - E. Pordomingo - A. Stramaglia (Eds.), Escuela y Literatura en Grecia Antigua, Cassino 2007, pp. 3966.

R. NiCOLAI, L'uso della storiografia come fonte di informazioni: teoria retorica e prassi oratoria, in Retórica e historiografía. El discurso militar en la historiografía desde la Antigüedad hasta el Rinacimiento, coord. por J.C. Iglesias Zoido, Madrid 2008, pp. 143-174.

A. NiCOLOSI, Riusi omerici nel primo 'epodo di Colonia' (Archil. fr. 196a $W^{2}$ ), Maia 57, 2005, pp. 243-259.

A. Nicolosi, Ipponatte, Epodi di Strasburgo. Archiloco, Epodi di Colonia. Con un'Appendice su P.Oxy. LXIX 4708, Bologna 2007.

A. Nicolosi, Su uno scolio marginale ad Hippon. fr. 88,2 Dg., ZPE 171, 2009, pp. 1-2.

A. NICOLOSI, Hippon. fr. ${ }^{\circ} 195 \mathrm{Dg} .\left(={ }^{*} 116 \mathrm{~W} .{ }^{2}\right)$ : marginalia, ZPE 174, 2010, pp. 15-18.

A. Nicolosi, P.Oxy. XXXVII 2811, fr. 5a-b rr. 9s. (= Adesp. Com. fr. ${ }^{*} 1114,66$ s. K.-A.), ZPE 177, 2011, pp. 27-29.

A. Nicolosi, P.Oxy. XVIII 2176 fr. 6,12 (= Hippon. fr. $131 \boldsymbol{E}$ $D g . ; 118$ D W. $\left.{ }^{2}\right)$, ZPE 180, 2012, pp. 49-50.

A. NicolOSI, Addendum et corrigendum ad ZPE 177, 2011, 2729 (Adesp. comic. fr. ${ }^{*} 1114,66$ s. K.-A.), ZPE 189, 2014, p. 64. 
NORSA-VITELLI 1934

NÜNLIST 2009

NÜNLIST 2012

NUTTON 1983

NutTON 1984

NutTON 1991

OBBINK 2003

OBBINK 2007

OLSON 2014

ORSINI 2005

PAAP 1948

Pacella 1986

PACK $1965^{2}$

PAgAni-Perrone 2012

PALMieri 1981

PALMIERI 1989

PALMIERI 2001

PARSONS 1982 $\triangle$ IHГHCEIC di poemi di Callimaco in un papiro di Tebtynis, a c. di M. NORSA-G. Vitelli, Firenze 1934.

R. NÜNLIST, The Ancient Critic at Work. Terms and Concepts of Literary Criticism in Greek Scholia, Cambridge 2009.

R. NÜNLIST, Topos didaskalikos and Anaphora: Two Interrelated Principles in Aristarchus' Commentaries, in Homer and the Bible in the Eyes of Ancient Interpreters, Ed. by M.R. Niehoff (Jerusalem Studies in Religion and Culture 16), LeidenBrill 2012, pp. 113-126.

V. Nutton, Rec. a Marganne 1981, MedHist 27, 1983, p. 97.

V. NutTON, Galen in the Eyes of his Contemporaries, BHM 58, 1984, pp. 305-314.

V. NuTTON, John of Alexandria again: Greek Medical Philosophy in Latin Translation, CQ 41, 1991, pp. 509-519.

4658. Prose on Star-Signs Quoting Homer, Hesiod, and Others, in The Oxyrhynchus Papyri, LXVIII, Ed. with Transl. and Notes by N. Gonis-D. OBBINK-P.J. Parsons, London 2003, pp. 52-72.

D. OвbinK, Readers and Intellectuals, in BOWMAN-ColesGonis-OBBink-PARSONs 2007, pp. 271-282.

S.D. OLSON, Eupolis frr. 326-497. Translation and Commentary (Fragmenta comica 8.3), Heidelberg 2014.

P. ORSINI, Manoscritti in maiuscola biblica. Materiali per un aggiornamento, Cassino 2005.

A.H.R.E. PAAP, De Herodoti reliquiis in papyris et membranis Aegyptiis servatis, Leiden 1948.

D. PACELla, 40. Herodotus I 115-16, in Papiri letterari greci della Bayerische Staatsbibliothek di Monaco di Baviera, II: Nr. 19-44, a c. di A. Carlini, Stuttgart 1986, pp. 89-91.

R.A. PACK, The Greek and Latin Literary Texts from GrecoRoman Egypt, Ann Arbor $1965^{2}$ (1952¹).

L. PAGANI-S. PerRONE, Le ekdoseis antiche di Omero nei papiri, in I papiri omerici, "Atti del convegno internazionale di studi", Firenze, 9-10 giugno 2011, a c. di G. Bastianini-A. Casanova, Firenze 2012, pp. 97-124.

N. PALMIERI, Un antico commento a Galeno della scuola medica di Ravenna, Physis 23, 1981, pp. 197-295.

N. PALMIERI, L'antica versione latina del «De sectis» di Galeno, Pisa 1989.

N. PALMIERI, Nouvelles remarques sur les commentaires à Galien de l'école médicale de Ravenne, in Docente natura, Mélanges de Médecine ancienne et Médiévale offerts à G. Sabbah, textes reunis par A. Debru-N. Palmieri, Saint-Etienne 2001, pp. 209-246.

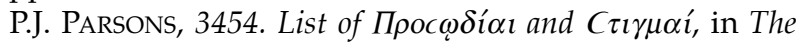
Oxyrhynchus Papyri, XLIX, Ed. with Transl. and Notes by A. Bülow-Jacobsen-J.E.G. Whitehorne, London 1982, pp. 97-98. 
PARSONS 2007

PAsQuali 1920

PASQuALi 1952

Pellé 2010

Pellé 2011

Pellé 2013

Pelling 2006

Pernigotti 2008

Perrone 2009a

Perrone 2009b

Perrotta 1938

Perrotta 1939

PfeIfFer 1926

PfeIfFer 1933

PfeIffer 1934

PfeIfFER 1949-1953

PfeIfFer 1968 (1973)

PHILIPPSON 1943

PINTAUdI 1994
P.J. PARsons, Copyists of Oxyrhynchus, in BOWMAN-COLESGonis-OBbink-PARSONs 2007, pp. 262-270.

G. PASQuali, Orazio lirico, Firenze 1920 [rist. con introd., indici ed aggiorn. bibliogr. a c. di A. La Penna, Firenze 1964].

G. PASQuAl, Storia della tradizione e critica del testo, Firenze $1952^{2}$ (1 $1^{\text {a }}$ ed. 1934).

N. PelLÉ, Chi, come e perché leggeva storiografia greca in Egitto, in Leggere greco e latino fuori dai confini nel Mondo Antico, "Atti del I Congresso Nazionale dell' Associazione Italiana di Cultura Classica", Lecce, 10-11 maggio 2008, a c. di M. Capasso, Lecce 2010, pp. 159-173.

N. Pellé, Le livre d'histoire dans les papyrus, Liège 2011.

N. Pellé, Libri scritture e scribi per i tre storici greci maggiori, Scripta 6, 2013, pp. 115-120.

C. PELLING, Homer and Herodotus, in Epic Interactions: Perspectives on Homer, Virgil, and the Epic Tradition, Ed. by M.J. Clarke-B.G.F. Currie-R.O.A.M. Lyne, Oxford 2006, pp. 75104.

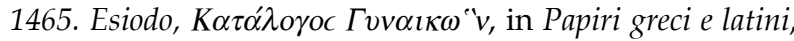
volume XV, a c. di V. Bartoletti-G. Bastianini-G. Messeri-F. Montanari-R. Pintaudi, Firenze 2008, pp. 32-33.

Commentaria et Lexica Graeca in Papyris reperta (CLGP), II Commentaria in adespota, 4 Comoedia et mimus, cur. S. PERRONE, Berlin 2009.

S. Perrone, Lost in Tradition. Papyrus Commentaries on Comedies and Tragedies of Unknown Authorship, TC 1, 2009, pp. 203-240.

G. PerRotTA, Il poeta degli epodi di Strasburgo, SIFC n.s. 15, 1938, pp. 3-41.

G. PerrotTA, Ancora gli epodi di Strasburgo, SIFC n.s. 16, 1939, pp. 177-188.

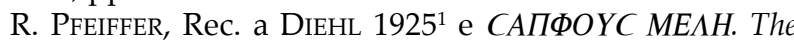
Fragments of the Lyrical Poems of Sappho, Ed. by E. Lobel, Oxford 1925, Gnomon 2, 1926, pp. 305-321.

R. PfeIfFER, Ein Epodenfragment aus dem Iambenbuche des Kallimachos, Philologus 88 n.F. 42, 1933, pp. 265-271.

R. PFEIFFER, Die neuen $\triangle I H \Gamma H C E I C$ zu Kallimachosgedichten, SBAW 10, 1934, pp. 1-50.

Callimachus, I Fragmenta, II Hymni et epigrammata, ed. R. PFEIFFER, Oxonii 1949-1953.

R. PfeIfFer, History of Classical Scholarship. From the Beginning to the End of the Hellenistic Age, Oxford 1968 [trad. it. Storia della filologia classica. Dalle origini alla fine dell'età ellenistica, introd. di M. Gigante, Napoli 1973].

R. Philippson, Papyrus Herculanensis 831, AJPh 64, 1943, pp. 148-162.

R. PINTAUd, Un frammento fiorentino (PL III/284 A) del POxy. II 231, Eirene 30, 1994, pp. 178-180. 
PIRROTTA 2009

Plezia 1949

PoethKe 2002

POLITO 1999

PORDOMINGO 2007

PORRO 2004

PORRO 2007

PORRO 2009

PORRO 2011

PORTER 1992

PÒRTULAS 1985

POTTER 1988

POWELl 1919

PRIESTLEy 2014

PriestLey-ZALi 2016

PRITCHET 1982

PRODI 2017

Puglia 1982

Puglia 1988

Puglia 1997
S. Pirrotta, Plato comicus: die fragmentarischen Komödien. Ein Kommentar, Berlin 2009.

M. PlezIA, De commentariis isagogicis, Polska Akademis Umiejetnosci, Archiwum Filologiczne Nr. 23, Kraków 1949.

A. PoethKe, Rec. a GMP, APF 48, 2002, pp. 180-182.

R. Polito, On the Life of Asclepiades of Bithynia, JHS 119, 1999, pp. 48-65.

F. PORDOMINGO, Ejercicios preliminares de la composición retórica y literaria en papiro: el encomio, in Escuela y Literatura en Grecia Antigua, "Actas del Simposio Internacional", Universidad de Salamanca (17-19 Noviembre de 2004), J.A. Fernández Delgado-F. Pordomingo-A. Stramaglia (eds.), Cassino 2007, pp. 405-453.

A. Porro, Alcaeus, in Commentaria et Lexica Graeca in Papyris reperta (CLGP) I.1.1, München-Leipzig 2004, pp. 75-246. A. Porro, Archiloco e gli Alessandrini, in La cultura letteraria ellenistica. Persistenza, innovazione, trasmissione, "Atti del convegno COFIN 2003", Università di Roma "Tor Vergata" (19-21 settembre 2005), a c. di R. Pretagostini-E. Dettori, Roma 2007, pp. 209-222.

A. PORRO, Forms and Genres of Alexandrian Exegesis on Lyric Poets, TC 1/2, 2009, pp. 183-202.

A. PORro, Archilochus, in Commentaria et Lexica Graeca in Papyris reperta (CLGP) I.1.3, München-Leipzig 2011, pp. 157-187.

J.I. PORTER, Hermeneutic Lines and Circles: Aristarchus and Crates on the Exegesis of Homer, in Homer's Ancient Readers: the Hermeneutics of Greek Epic's Earliest Exegetes , Ed. by R. Lamberton-J.J. Keaney, Princeton 1992, pp. 67-114.

J. PÒRTULAS, Ipponatte e Petronio, QUCC 48 n.s. 19, 1985, pp. 121-139.

P. PotTer, Hippocrates, V e VI, Cambridge (Mass.) 1988.

J.U. Powell, On the New Fragments of Greek Poetry Recently Published at Berlin, CR 33, 1919, pp. 90-91.

J. PRIESTLEY, Herodotus and Hellenistic Culture. Literary Studies in the Reception of the Histories, Oxford 2014.

Brill's Companion to the Reception of Herodotus in Antiquity and Beyond, Ed. by J. PRIESTLEY-V. ZALI (Brill's Companion to Classical Reception 6), Leiden-Boston 2016.

C.D. PRITCHET, Iohannis Alexandrini commentaria in librum De sectis Galeni, Leiden 1982.

E.E. Prodi, P.Oxy. 2174 fr. 5: An Odyssey for Hipponax, APF 63, 2017, pp. 1-10.

E. Puglia, La filologia degli Epicurei, CErc 12, 1982, 19-34.

E. Puglia, Aporie testuali ed esegetiche in Epicuro (PHerc.1012) (La scuola di Epicuro 8), Napoli 1988.

E. Puglia, La cura del libro nel mondo antico. Guasti e restauri del rotolo di papiro, Napoli 1997. 
RADERMACHER 1902

RADT 1977

RAMELLI 2004

RANKE 1840

RAWSON 1982

REA 1974

REDONDO 1990

RegALi 2007

REITER 2003

REITZENSTEIN 1897

REITZENSTEIN 1899

RENGAKOS 2006

RENNER 1978

RENNER 1997

REYNOLDS-WILSON 1968

RICHARD 1950

RISPOLI 1985

ROBERTS 1938

ROBERTS 1950

ROBERTS 1954

ROBERTS 1956
L. RADERMACHER, Aus dem zweiten Bande der Amherst Papyri, RhM N.F. 57, 1902, pp. 137-151.

Tragicorum Graecorum Fragmenta (TrGF), vol. 4: Sophocles, ed. S. RADT, Göttingen 1977.

I. RAMELLI, Allegoria: I, L'età classica, Milano 2004.

C.F. RANKE, Hesiodi quod fertur Scutum Herculis, Quedlinburg-Leipzig 1840.

E. RAWSON, The Life and Death of Asclepiades of Bithynia, CQ 32, 1982, pp. 358-370.

J.R. REA in An Exhibition of Papyri, mainly in Greek, at the Bodleian Library and at the Ashmolean Museum, for the XIV International Congress of Papyrologists, Oxford, 24-31 July 1974, Oxford 1974.

E. REDONDO, Dos notas sobre poetas yámbicos, Veleia 7, 1990, pp. 251-264.

M. RegALI, s.v. Minucius Pacatus Irenaeus, in LGGA, 2007.

F. REITER, Rec. a MARGANNE 1998a, BO 60, 2003, pp. 378389.

R. REITZENSTEIN, Geschichte der griechischen Etymologica, Leipzig 1897.

R. REITZENSTEIN, Zwei neue Fragmente der Epoden des Archilochos, SPAW 44-45-46, 1899, pp. 857-864.

A. RENGAKOS, Homer and the Historians: The Influence of Epic Narrative Technique on Herodotus and Thucydides, in La poésie épique grecque: métamorphoses d'un genre littéraire, préparés et présidés par F. Montanari-A. Rengakos (Entretiens sur l'Antiquité classique 52), Genève-Vandœuvres 2006, pp. 183-209.

T. RENNER, Four Michigan Papyri of Classical Greek Authors, ZPE 29, 1978, pp. 5-26.

T. RENNER, Towards Plato in Context: A Papyrus Containing Phaedo 99A-100B from CS 190 (B 224?) at Karanis, in "Akten des 21. Internationalen Papyrologenkongresses", Berlin 13.-19.8.1995, hrsg. von B. Kramer et al., Stuttgart-Leipzig 1997, II, pp. 827-834.

L.D. ReYNOLDS-N.G. WILSON, Scribes and Scholars, Oxford 1968 (trad. it. Copisti e Filologi. La tradizione dei classici dall'antichità ai tempi moderni, Padova 1987³).

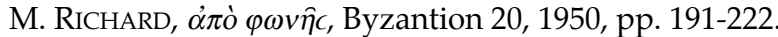

G.M. RisPOLI, L'artista sapiente. Per una storia della fantasia, Napoli 1985.

Catalogue of the Greek and Latin Papyri in the John Rylands Library, Manchester. III, Theological and Literary Texts, Ed. by C.H. ROBERTS, Manchester 1938.

The Antinoopolis Papyri, I, Ed. with Transl. and Notes by C.H. ROBERTS, London 1950.

C.H. RoBERTS, The Codex, PBA 40, 1954, pp. 184-185.

C.H. RoBERTS, Greek Literary Hands (350 B.C.-A.D. 400), Oxford 1956. 
ROSELLI 1988

RoselLi 1990

ROSELLI 1998

RoselLi 2012a

ROSELLI 2012b

RosÉN 1962

RosÉN 1987

RosÉN 1997

Rosen 1988

Rosen 1990

VAN ROSSUM-STEENBEEK 1998

ROSTAGNI 1935

ROUECHÉ 1999

RUDBERG 1941

Russo 1965

RZACH 1898

SAERENS 1990

SANDBACH 1967

SANDBACH 1969

SANDERS 1999

SCARBOROUGH 1981

SCARBOROUGH 1987
A. Roselli, Citazioni ippocratiche in Demetrio Lacone (PHerc. 1012), CErc 18, 1988, pp. 53-57.

A. Roselli, Appunti per una storia dell'uso apologetico della filologia: la nuova edizione di Demetrio Lacone (PHerc. 1012), SCO 40, 1990, pp. 117-138.

A. Roselli, L'Anonimo De medicina (II 244-245 Dietz): un prolegomenon alla lettura di testi medici? FAM 15, 1998, pp. 7-23.

A. Roselli, Galeno e la filologia del II secolo, in Vestigia notitiai, Scritti in memoria di Michelangelo Giusta, a c. di E. Bona-C. Levy-G. Magnaldi, Alessandria 2012, pp. 63-80.

A. Roselli, Galeno e le edizioni ippocratiche di Artemidoro Capitone e Dioscoride, in Sulla tradizione indiretta dei testi medici greci, "Atti del IV seminario internazionale di Siena", Certosa di Pontignano, 3-4 giugno 2011, a c. di S. Fortuna-I. Garofalo-A. Lami-A. Roselli, Pisa 2012, pp. 15-27.

H.B. RoséN, Eine Laut- und Formenlehre der Herodotischen Sprachform, Heidelberg 1962 (Lexeis erodotee: pp. 222-231). Herodotus. Historiae, I, libros I-IV continens, ed. H.B. RosÉN, Leipzig 1987.

Herodotus. Historiae, II, libros V-IX continens, ed. H.B. Rosén, Stutgardiae et Lipsiae 1997.

R.M. Rosen, Hipponax, Boupalos, and the Conventions of the psogos, TAPhA 118, 1988, pp. 29-41.

R.M. Rosen, Hipponax and the Homeric Odysseus, Eikasmós 1, 1990, pp. 11-25.

M. van Rossum-SteEnBEeK, Greek Raeders' Digests? Studies on a Selection of Subliterary Papyri, Leiden-New York-Köln 1998. A Rostagni, Rec. a Pfeiffer 1934, Gnomon 11, 1935, pp. 366-368.

M. Roueché, Did Medical Students Study Philosophy in Alexandria?, BICS 43, 1999, pp. 153-169.

G. RudBERG, Herodotea. I. De papyro Oxyrhynch. 1092 adnotationes, Eranos 39, 1941, pp. 145-147.

C.F. Russo, Hesiodi Scutum, Firenze $1965^{2}$.

A. RZACH, Die handschriftliche Tradition der pseudohesiodischen Aspis, Hermes 33, 1898, pp. 591-625.

C. SAERENS, Papyrus d'Hérodote et tradition manuscrite, in Studia varia Bruxellensia ad orbem Graeco-Latinum pertinentia, II, ed. R. De Smet-H. Melaerts-C. Saerens, Leuven 1990, pp. 177-192.

F.H. SANDBACH, Plutarchi Moralia, vii, Leipzig 1967.

F.H. SAndBach, Plutarch's Moralia, xv: Fragments, Cambridge (Mass.) 1969.

K.J. SANDERS, Toward a New Edition of PHerc. 831, CErc 29, 1999, pp. 17-30.

J. SCARBOROUGH, The Galenic Question, ZWG 45, 1981, pp. $1-30$

J. SCARborough, Rec. a CAVINI-DONNINI MaCciò-FunGHIMANETTI 1985, Isis 78, 1987, p. 298. 
SCHIRONI 2010

SCHIRONI 2012

SCHMIDT 1854

SCHMIDT 1912

SCHMIDT 1976

SCHNEIDEWIN 1839

SCHÖNE 1905

SCHROEDER 2006

SCHROEDER 2007

SCHUBART 1907

SCHUBART 1918

SCHUBART 1950

SCHUBART-Wilamowitz 1907 B. P. 9773, in Lyrische und dramatische Fragmente, bearb. von W. SCHUBART - U. vON WILAMOWITZ-MOELLENDORFF, Berlin 1907, pp. 129-130.

SCHULTZ 1910

SCHULTZ 1913

SEIDER 1967-1970

SETAIOLI 1988

SITZLER 1919

F. SCHIRONI, Tò $\mu \varepsilon ́ \gamma \alpha \beta \imath \beta \lambda i ́ o v$. Book-ends, End-titles, and Coronides in Papyri with Hexametric Poetry, Durham (NC) 2010. F. SCHIRONI, Greek Commentaries, Dead Sea Discoveries 19, 2012, pp. 399-441.

Didymi Chalcenteri grammatici Alexandrini fragmenta quae supersunt omnia, ed. M. SCHMIDT, Leipzig 1854.

K.F.W. SchMidT, Rec. a P.Oxy. VIII, GGA 174, 1912, pp. 633652.

M. SCHMIDT, Die Erklärungen zum Weltbild Homers und zur Kultur der Heroenzeit in den bT-Scholien zur Ilias, Munich 1976.

Delectus poesis Graecorum elegiacae, iambicae, melicae, ed. F.G. SCHNEIDEWIN, Sectio II et III, Gottingae 1839.

Medizinische und naturawissenschaftlichen Texte, in Griechische Papyri medizinischen und naturawissenschaftlichen Inhalts, berab. von K. Kalbfleisch-H. SCHÖNE (BKT III), Berlin 1905, pp. 22-26

C.M. SCHROEDER, Hesiod in the Hellenistic Imagination, U. Mich. Diss., 2006.

C.M. SCHROEDER, A New Monograph by Aristarchus?, JHS 127, 2007, pp. 138-141.

W. SCHUBART, Das Buch bei den Griechen und Römern, Berlin 1907 (Leipzig 1921², 19623).

W. SCHUBART, Einführung in die Papyruskunde, Berlin 1918. 10. Episches Gedicht, in W. SCHUBART, Griechische literarische papyri, Berlin 1950, pp. 26-28.

H. SCHULTZ, Die handschriftliche Überlieferung der HesiodScholien, in Abhandlungen der königlichen Gesellschaft der Wissenschaftern zu Göttingen, Philol.-Hist. K1. 12/4, 1910, pp. VIII-101.

H. SCHULTZ, Zu Nebenüberlieferung der Hesiodscholien, in Nachrichten von der königlichen Gesellschaft der Wissenschaftern zu Göttingen, Philol.-Hist. Kl., 1913, pp. 252-263.

SCHWYZER-Debrunner 1950 E. SCHWYZER-A. DebrunNer, Griechische Grammatik auf der Grundlage von Karl Brugmanns Griechischer Grammatik, II: Syntax und syntaktische Stilistik, München 1950.

R. SEIDER, Paläographie der griechischen Papyri, I-II, Stuttgart 1967-1970.

A. SetaIOl, Episodi erodotei nell'opera senecana. Dalla narrazione all'exemplum, in A. Setaioli, Seneca e i Greci. Citazioni e traduzioni nelle opere filosofiche, Bologna 1988, pp. 485-503 (= Dalla narrazione all'exemplum. Episodi erodotei nell'opera senecana, in Letterature classiche e narratologia, "Atti del Convegno Internazionale", Selva di Fasano (Brindisi) 6-8 ottobre 1980, Perugia 1981, pp. 379-396).

J. SITZLER, Jahresbericht über die griechischen Lyriker (mit Aus- 
SITZLER 1922

SLATER 1986

SLINGS 1987

SNELL 1929

SNELL 1939

SNELL 1971

SNELL 1972

SNODGRASS 1964

SOLDATI 2005

SOLMSEN 1965

SOLMSEN 1990

STEIN 1871

STROPPA 2008a

STROPPA 2008b

SUÁREZ DE LA TORRE 2002

TAILLARDAT 1967

TAMMARO 1988

TARDITI 1968

TEMKIN 1932

TEMKIN 1935

TeRZAGHI 1940 schluß Pindars), sowie über die Bukoliker und die Anthologia Palatina für 1905-1917, JAW 174, 1919, p. 37.

J. SITZLER, Bericht über Herodot, 1915-1920, JAW 191, 1922, pp. 1-25.

Aristophanis Byzantii fragmenta, post A. Nauck collegit, testimoniis ornavit, brevi commentario instruxit W.J. SLATER, Berlin-New York 1986.

S.R. SLINGS, D. Anonymus. Commentary on Poem(s) of Hipponax, in Some Recently Found Greek Poems, Text and Comm. by J.M. Bremer-A.M. van Erp Taalman Kip-S.R. Slings, Leiden-New York-København-Köln 1987, pp. 70-94.

B. SNELL, Bericht über Herodot, 1921-1927, JAW 220, 1929, pp. 1-36.

B. SNELL, Rec. a P.Mil.Vogl. I e P.Ryl. III, Gnomon 15, 1939, p. 543.

Tragicorum Graecorum Fragmenta (TrGF), 1: Didascaliae tragicae; Catalogi tragicorum et tragoediarum; testimonia et fragmenta tragicorum minorum, ed. B. SNELL, Göttingen 1971.

Frügriechische Lyriker, 2. Die Jambographen, deutsch von Z. Franyó, griechischer Text bearb. von B. SNELL, Berlin 1972 $\left(1981^{2}\right)$.

A.M. SNODGRASS, Carian Armourers. The Growth of a Tradition, JHS 84, 1964, pp. 107-118.

A. Soldati, Due frammenti di un unico rotolo? P.Duke inv. 756 e P.Mil.Vogl. inv. 1358 (Herodotus IV 144.2-145.1 e 147.4-5), BASP 42, 2005, pp. 101-106.

F. SOLMSEN, Ilias $\Sigma$ 535-540, Hermes 93, 1965, pp. 1-6.

Hesiodi Theogonia, Opera et Dies, Scutum, ed. F. SolmSEN, Oxford $1990^{3}$.

Herodoti Historiae, recensuit H. STEIN, II, Berlin 1871.

M. STROPPA, Esegesi a Esiodo nei papiri, in BASTIANINI-CASANOVA 2008, pp. 83-95.

M. STROPPA, Lista di codici tardoantichi contenenti hypomnemata, Aegyptus 88, 2008, pp. 49-69.

Yambógrafos Griegos, introd., trad. y notas de E. SUÁREZ DE LA TORRE, Madrid 2002.

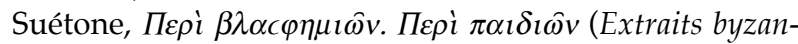
tins), par J. TAILLARDAT, Paris 1967.

V. TAMMARO, Spergiuri e naufragi, GFF 11, 1988, pp. 57-58. Archilochus, fragmenta ed., veterum testimonia coll. I. TARDITI, Romae 1968.

O. TEMKIN, Geschichte des Hippokratismus im Ausgehenden Altertum, Kyklos 4, 1932, pp. 1-80 (= History of Hippocratism in Late Antiquity, in The double Face of Janus, Baltimore 1977, pp. 167-177).

O. TeMKIN, Studies on Late Alexandrian Medicine: Alexandrian Commentaries on Galen's De sectis ad introducendos, BHM 3, 1935, pp. 403-430.

N. TerZAghi, L'odio di Ipponatte ed il primo epodo di Stra- 
THEODORIDIS 1986

VAN THIEL 2014

TosI 1988

TOSI 1994

TRABATTONI 1996

TREU 1959

TRIBULATO 2016

TROJAHN 2002

TSANTSANOGLOU 2010

TURNER 1952

TURNER 1956

TURNER 1962

TURNER 1977

TURNER $1980^{2}$

TURNER-PARSONS 1987

UCCIARDELLO 2005

UCCIARDELLO 2006

UCCIARDELLO 2007

UCCIARDELLO 2012

UEBEL 1971

Uebel 1976

VAN DER VALK 1949 sburgo, SIFC 17, 1940, pp. 217-235 [rist. in Studia Graeca et Latina, I, Torino 1963, pp. 314-332].

C. THEODORIDIs, Ein neues Testimonium für Hipponax fr. 73,3 Degani, Hermes 114, 1986, pp. 374-375.

H. van THIEL, Aristarch, Aristophanes Byzantios, Demetrios Ixion, Zenodot. Fragmente zur Ilias gesammelt, neu herausgegeben und kommentiert, I-IV, Berlin-Boston 2014.

R. TosI, Studi sulla tradizione indiretta dei classici greci, Bologna 1988.

R. Tosi, La lessicografia e la paremiografia in età alessandrina e il loro sviluppo successivo, in MONTANARI 1994, pp. 143-209. F. TRABATTONI , Rec. a CPF III, GCFI 16, 1996, pp. 118-121. Archilochos, ed. M. TREU, München 1959.

O. Tribulato, Herodotus' Reception in Ancient Greek Lexicography and Grammar: From the Hellenistic to the Imperial Age, in PRIESTLEY-ZaLi 2016, pp. 169-192.

S. Trojahn, Die auf Papyri erhaltenen Kommentare zur Alten Komödie. Ein Beitrag zur Geschichte der antiken Philologie, München-Leipzig 2002.

K. Tsantsanoglou, Hipponactea, Eikasmós 21, 2010, pp. 1528.

E.G. TURNER, Roman Oxyrhynchus, JEA 38, 1952, pp. 78-93 [rist. in BOWMAN-COLES-GONIS-OBBinK-PARSONS 2007, pp. 141-154].

E.G. TURNER, Scribes and Scholars of Oxyrhynchus, in "Akten des VIII. Internationalen Kongresses für Papyrologie" (Wien 1955), MPER N.S. 5, Wien 1956, pp. 141-146 (rist. in BOWMAN-COLES-GONIS-OBBINK-PARSONS 2007, pp. 256-261). E.G. TURNER, L'érudition alexandrine et les papyrus, CE 37, 1962, pp. 135-152.

E.G. TURnER, The Typology of the Early Codex, Philadelphia 1977.

E.G. Turner, Greek Papyri. An Introduction, Oxford $1980^{2}$ $\left(1968^{1}\right)$ [trad. it. Papri greci, a c. di M. Manfredi, Roma 1984 $\left.\left(2002^{2}\right)\right]$.

Greek Manuscript of the Ancient World, by E.G. TuRNER, Second Ed. Rev. and Enl., Ed. by P.J. PARSONS, London 1987.

G. UCCIARDELLO, s.v. Sal(l)ustius [2], in LGGA, 2005.

G. UcCiardello, s.v. Claudius [2] Casilo, in LGGA, 2006.

G. UCCIARDELlo, s.v. Philemon [3], in LGGA, 2007.

G. UCCIARDELlo, 11. Frammento epodico (Archiloco?), in $\mathrm{Li}$ terarische Texte der Berliner Papyrussammlung, hrsg. von F. Reiter, Berlin 2012, pp. 60-76.

F. UEBEL, Literarische Texte unter Ausschluss der christlichen, APF 21, 1971, pp. 167-206.

F. UEBEL, Literarische Texte unter Ausschluss der christlichen, APF 24-25, 1976, pp. 191-251.

M. VAN DER VALK, Textual Criticism of the Odyssey, Leiden 1949. 
VANNINI 2007

VANNINI 2009a

VANNINI 2009B

VIERECK 1902

VILJOEN 1915

VISENTINI 2012-2013

VITELLI 1929a

Vitelli 1929b

VOGLIANO 1937

VOGLIANO 1948

VON STADEN 2006

VON STADEN 2009

WATSON 1991

WATSON 2005

WELCKER 1817

West 1966

WEST 1974

WeST 1978

West 1980

WEST 1983

West 1985

WEST $1989^{2}$

West 2001
L. VANNINI, Un commentario a Pindaro: PSI XIV 1391 con nuovi frammenti, in Comunicazioni dell'Istituto Papirologico «G. Vitelli» 7, Firenze 2007, pp. 29-73.

L. VANNINI, Le tipologie dell'esegesi antica testimoniate nei papiri, Firenze (Diss.) 2009.

L. VANNINI, Nuove letture in P.Amh. II 12, commentario a Erodoto I, in Comunicazioni dell'Istituto Papirologico «G. Vitelli» 8, Firenze 2009, pp. 93-101.

P. VIERECK, Rec. a P.Amh. II, BPhW 22, 1902, coll. 715-720. H.G. VILJOEN, Herodoti fragmenta in papyris servata, Groningen 1915.

S. VISENTINI, Problemi di trasmissione e ricezione della letteratura greca nei 'papiri scolastici' di età ellenistica e romana, Trieste (Diss.) 2012-2013.

G. VITELLI, Da papiri della società italiana. I. Scolii a Giambi di Callimaco, BSAA 24, 1929, pp. 1-4.

1094. Frammenti di scolii a Giambi di Callimaco, in Papiri greci e latini, IX, Firenze 1929, pp. 157-164.

18. $\triangle$ IHГHCEIC di poemi di Callimaco, in Papiri della R. Università di Milano, I, ed. da A. Vogliano, Milano 1937, pp. 66-145.

A. Vogliano, Nota Hipponattea, Acme 1, 1948, pp. 257-259. $\mathrm{H}$. VON STADEN, Interpreting "Hippocrates" in the $3 \mathrm{rd}$ and 2 nd Centuries BC, in Ärzte und ihre Interpreten, hrsg. von C.W. Müller et al., München-Leipzig 2006, pp. 15-47.

H. vON StADEN, Staging the Past, Staging Oneself: Galen on Hellenistic Exegetical Traditions, in Galen and the World of Konowledge, Ed. by C. Gill et al., Cambridge 2009, pp. 132-156.

L.C. WATson, Arae. The Course Poetry of Antiquity, Leeds 1991.

L.C. Watson, A Commentary on Horace's Epodes, Oxford 2005.

Hipponactis et Ananii iambographorum fragmenta coll. et rec. F.T. WELCKER, Gottingae 1817.

Hesiod Theogony, Ed. with prolegomena and comm. by M.L. WEST, Oxford 1966.

M.L. West, Studies in Greek Elegy and Iambus, Berlin-New York 1974.

Hesiod Works and Days, Ed. with prolegomena and comm. by M.L. WEST, Oxford 1978.

M.L. WeSt, Delectus ex iambis et elegis Graecis, Oxonii 1980.

M.L. West, The Hesiodic Catalogue: Xouthids and Aiolids, ZPE 53, 1983, pp. 27-30.

M.L. WeSt, The Hesiodic Catalogue of Women: Its Nature, Structure, and Origins, Oxford 1985.

Iambi et elegi Graeci ante Alexandrum cantati, ed. M.L. WEST, I, Oxonii $1989^{2}\left(1971^{1}\right)$.

M.L. WEST, Studies in the text and transmission of the Iliad, München-Leipzig 2001. 
West 2008

WEST 1967

WEST 2007

West 2011

WESTERINK 1964

WESTERINK 1981

WILAMOWITZ 1900

WILAMOWITZ 1918

WILAMOWITZ 1924

WILSON 1983

WILSON 1984

WILSON 2015a

WILSON 2015b

WINTER 1925

WITTERN 1972

WOLSKA-CONUS 1992

WOUTERS 1977

WYSS 1936
M.L. WeSt, The Hesiod Papyri and the Archaic Epic Language, in BASTIANINI-CASANOVA 2008, pp. 29-42.

The Ptolemaic Papyri of Homer, Ed. by S. WEST, KölnOpladen 1967.

S.R. WeSt, The Text, I. Transmission and Ancient Reception, in Herodotus, Histories, Book VIII, Ed. by A.M. Bowie, Cambridge 2007, pp. 30-34.

S.R. West, The Papyri of Herodotus, in Culture in Pieces. Essays on Ancient Texts in Honour of Peter Parsons, Ed. by D. Obbink-R. Rutherford, Oxford 2011, pp. 69-83.

L.G. WesterinK, Philosophy and Medicine in Late Antiquity, Janus 51, 1964, pp. 169-177.

L.G. WesterinK, Agnellus of Ravenna. Lectures on Galen' de sectis (Seminar Classics 609, Arethusa Monographs VIII), State University of New York at Buffalo 1981.

U. VON WILAMOWITZ-MOELLENDORFF, Textgeschichte der griechischen Lyriker, Berlin 1900.

U. VON WILAMOWITZ-MOELLENDORFF, 3. Homerische Glossen, SPAW 32, 1918, pp. 739-742.

U. VON WILAMOWITZ-MOELLENDORFF, Lesefrüchte, Hermes 59, 1924, pp. 249-273 [= Kleine Schriften, IV Lesefrüchte und Verwandtes, Berlin 1962, pp. 343-367].

N. WILSON, Scholars of Byzantium, London 1983 (trad. it. Filologi Bizantini, Napoli 1989).

N.G. WiLsON, The Relation of Text and Commentary in Greek Books, in Il libro e il testo, "Atti del Convegno Internazionale (Urbino, 20-23 settembre 1982)”, a c. di C. Questa-R. Raffaelli, Urbino 1984, pp. 103-110.

Herodoti Historiae, ed. N.G. WILSON, Oxford 2015.

N.G. WILSON, Herodotea. Studies on the Text of Herodotus, Oxford 2015.

J.G. WINTER, A Fragment of Demosthenes' Third Philippic in the University of Michigan Collection, CPh 20, 1925, pp. 97114.

R. WITTERN, Die hippokratische Schrift De morbis I, Hildesheim-New York 1972.

W. Wolska-Conus, Les commentaires de Stéphanos d'Athènes aux Prognostikon et aux Aphorismes d'Hippocrate: de Galièn à la pratique scolaire alexandrine, REByz 50, 1992, pp. 5-86.

A. Wouters, Hermann Diels und P. Oxy. 3.437, Philologus 121, 1977, pp. 146-149.

Antimachi Colophonii reliquiae, coll., disp., explic. B. WYSS, Berolini 1936. 